\title{
IL-6 mediates platinum-induced enrichment of ovarian cancer stem cells
}

\author{
Yinu Wang, ${ }^{1,2}$ Xingyue Zong, ${ }^{1}$ Sumegha Mitra, ${ }^{3}$ Anirban Kumar Mitra, ${ }^{1,4,5}$ Daniela Matei, ${ }^{2}$ \\ and Kenneth P. Nephew ${ }^{1,4,6}$ \\ 'Medical Sciences, Cell, Molecular and Cancer Biology Program, Indiana University School of Medicine, Indiana University \\ Bloomington (IUB), Bloomington, Indiana, USA. ${ }^{2}$ Department of Obstetrics and Gynecology, Northwestern University \\ Feinberg School of Medicine, Chicago, Illinois, USA. ${ }^{3}$ Department of Obstetrics and Gynecology, Indiana University \\ School of Medicine, ${ }^{4}$ Indiana University Melvin and Bren Simon Cancer Center, and ${ }^{5}$ Medical and Molecular Genetics, \\ Indiana University School of Medicine, Indiana University, Indianapolis, Indiana, USA. ${ }^{6}$ Department of Cellular and \\ Integrative Physiology Indiana University School of Medicine, Indianapolis, Indiana, USA.
}

In high-grade serous ovarian cancer (OC), chemotherapy eliminates the majority of tumor cells, leaving behind residual tumors enriched in OC stem cells (OCSC). OCSC, defined as aldehyde dehydrogenase-positive $\left(\mathrm{ALDH}^{+}\right)$, persist and contribute to tumor relapse. Inflammatory cytokine IL-6 is elevated in residual tumors after platinum treatment, and we hypothesized that IL-6 plays a critical role in platinum-induced OCSC enrichment. We demonstrate that IL-6 regulates stemness features of OCSC driven by ALDH1A1 expression and activity. We show that platinum induces IL-6 secretion by cancer-associated fibroblasts in the tumor microenvironment, promoting OCSC enrichment in residual tumors after chemotherapy. By activating STAT3 and upregulating ALDH1A1 expression, IL-6 treatment converted non-OCSC to OCSC. Having previously shown altered DNA methylation in OCSC, we show here that IL-6 induces DNA methyltransferase 1 (DNMT1) expression and the hypomethylating agent (HMA) guadecitabine induced differentiation of OCSC and reduced - but did not completely eradicate - OCSC. IL-6 neutralizing antibody (IL-6-Nab) combined with HMA fully eradicated OCSC, and the combination blocked IL-6/IL6-R/pSTAT3-mediated ALDH1A1 expression and eliminated OCSC in residual tumors that persisted in vivo after chemotherapy. We conclude that IL-6 signaling blockade combined with an HMA can eliminate OCSC after platinum treatment, supporting this strategy to prevent tumor recurrence after standard chemotherapy.

Authorship note: $X Z$ and SM are co-second authors.

Conflict of interest: The authors have declared that no conflict of interest exists.

License: Copyright 2018, American Society for Clinical Investigation.

Submitted: May 21, 2018

Accepted: October 29, 2018

Published: December 6, 2018

Reference information: JCI Insight. 2018;3(23):e122360. https://doi.org/10.1172/jci. insight. 122360 .

\section{Introduction}

Epithelial ovarian cancer (OC) is considered a chemoresponsive tumor with high initial response rates to standard therapy consisting of platinum/paclitaxel (1). However, most women with high-grade serous carcinoma (HGSC) eventually develop recurrence, which rapidly evolves into chemoresistant disease (2). Recurrent OC is incurable; therefore, novel strategies to prevent disease relapse and chemoresistance are needed.

It has been hypothesized that chemoresistant cancers are driven by cancer stem cells (CSCs) (3). Although chemotherapy effectively decreases tumor burden, it also contributes to enriching the population of OC stem cells (OCSC) in residual tumors (4), supporting the concept that OCSC contribute to tumor relapse after therapy. OCSC are characterized by expression of specific cell surface markers (4-6) and the ability to self-renew, differentiate, and generate tumors when injected in small numbers (50-1,000 cells) in NOD/SCID mice (7). The most robust marker currently used to identify CSCs in ovarian tumors, as well as other solid tumors, remains aldehyde dehydrogenase (ALDH) activity (6). $\mathrm{ALDH}^{+}$cells isolated from HGSC cell lines and primary human tumors $(4,6)$ displayed stem cell properties, including spheroid-forming and tumor-initiating capacity, enhanced drug resistance, upregulation of stemness-associated transcription factors (e.g., Sox2, Nanog, Oct4), and expression and corresponding downregulation of the expression of differentiation-related genes HOXA10 and HOXA11 $(4,6)$. As a member of the ALDH family of detoxifying enzymes (8), ALDH1A1 has also been proposed as a functional regulator of OCSC. ALDH1A has been shown to be essential for oxidation of intracellular aldehydes (8) and is reported to play a key role in early differentiation of stem cells through oxidation of retinol to retinoic acid (9). Furthermore, therapies targeting ALDH1A1 appear to be a promising approach for eradicating CSCs and preventing chemoresistant tumor relapse (4). 
However, it has been recently recognized that differentiated cancer cells can acquire self-renewal and stemness properties under the influence of extrinsic factors found in the tumor microenvironment (TME) (10). Proinflammatory factors in the TME recently reported to play a regulatory role in CSC proliferation include IL-1, -6, and -23 (11) and the transcription factor NF- $\mathrm{kB}$ (12). IL-6, a cytokine that stimulates cell proliferation and invasion, is enriched in OC-associated malignant ascites (12-14). Cancer associated fibroblasts (CAFs) in the ovarian TME serve as a reservoir for protumorigenic inflammatory cytokines, including IL-6 $(15,16)$. It has been demonstrated that CAF-cancer cell crosstalk plays a key role in OC progression (17), maintaining an optimal microenvironment for OC cell survival and proliferation. Furthermore, platinum-DNA damage induced secretion of IL- 6 by OC cells and contributed to chemoresistance (18), suggesting an important connection between platinum activation of the IL- 6 signaling pathway and OC progression. In this regard, IL-6 has been hypothesized to create a protective niche, maintaining survival of residual tumor cells and consequently contributing to tumor relapse (16).

Epigenetic dysregulation that results from the reciprocal interplay between immune, stromal, and cancer cells plays a pivotal role in driving tumor initiation and tumor progression (19-22). Crosstalk between tumor cells and the microenvironment is mediated by both cell-to-cell contact and soluble substances, leading epigenetic alterations in both neoplastic and the surrounding nontumorigenic cells, including CAFs, and contributing to the formation of a "cancer favorable niche" (19-21, 23). Extensive studies highlight that the epigenetic effects of chronic inflammation and immune cells on tumor cells to increase tumorigenesis risk. Inflammation cytokine IL-6, in the context of gastric cancer and colon cancer, induced upregulation of DNA methyltransferase 1 (DNMT1), leading to DNMT-mediated gene silencing and tumorigenesis (19, 24, 25). Altered DNA methylation has been associated with CSC phenotype maintenance (4) and has been linked to the undifferentiated phenotype of CSCs. We demonstrated that hypomethylating agents (e.g., guadecitabine, decitabine) inhibit stemness characteristics and tumor initiating capacity (4). In this regard, blocking IL-6 signaling in combination with a hypomethylating agent (HMA) may be a promising approach to disrupt crosstalk between tumor cells and their protective niche and to target OCSC. Early clinical trials using antibodies against human IL-6 (Siltuximab) or IL-6 receptor (IL-6R) (Tocilizumab) reported some activity as single agents (26), but convincing clinical activity has not yet been demonstrated (27), suggesting that rationally designed combinations should be investigated.

Here, we demonstrate that treatment of OC cells with platinum- or IL-6-induced pSTAT3 signaling, which upregulated ALDH1A1 expression, increased stemness-associated genes and DNMT1 and enriched the population of $\mathrm{ALDH}^{+}$cells. These cells displayed enhanced spheroid formation ability and increased resistance to platinum. Functional consequences of these molecular and cellular changes were further investigated using an in vivo model enriched in CSCs after platinum treatment. OCSC were targeted with an IL-6 neutralizing antibody $(\mathrm{Nab})$ combined with the second-generation HMA guadecitabine. The combination treatment inhibited the stemness features of tumor cells persisting after chemotherapy and eradicated the $\mathrm{ALDH}^{+}$population. These results support a combination between an epigenetic modulator and an anti-IL-6 antibody as a potentially novel strategy following chemotherapy with the goal of targeting surviving OCSC and preventing disease recurrence.

\section{Results}

IL-6 expression, OC progression, and reduced chemotherapy response. Inflammatory responses including IL-6mediated inflammation have been shown to contribute to OC progression and chemoresistance (12). Analysis of the transcriptomic profiles of high-grade serous ovarian cancer (HGSOC) tumors from The Cancer Genome Atlas (TCGA) data portal demonstrated that upregulation of IL-6 expression was significantly associated with poor ( $<12$ months) tumor-free survival (Figure 1A). Moreover, high IL-6 expression correlated $(P=0.0023)$ with reduced progression-free survival after initial chemotherapy (Figure $1 \mathrm{~B})$. In a panel of OC cell lines, we examined expression of IL- 6 and IL-6R, IL- 6 secretion in vitro by ELISA, and IL-6 levels after treatment with an $\mathrm{IC}_{50}$ dose of cisplatin (CDDP; Supplemental Table 1; supplemental material available online with this article; https://doi.org/10.1172/jci.insight.122360DS1). Levels of mRNA for IL-6 and IL-6R and basal IL-6 levels in conditioned media (CM) varied among the OC cell lines (Figure 2, A-D); however, CDDP treatment consistently increased $(P<0.01)$ IL-6 levels in CM (Figure 2D).

We further examined the association between increased IL-6, response to platinum treatment, and OC progression in vivo using an intraperitoneal xenograft model derived from platinum-sensitive A2780 OC cells. Mice were treated with vehicle or carboplatin $(50 \mathrm{mg} / \mathrm{ml}$ weekly for 3 weeks), and blood and residual tumors were collected at sacrifice (day 21). Plasma IL-6 levels in carboplatin-treated mice were increased ( $P$ 

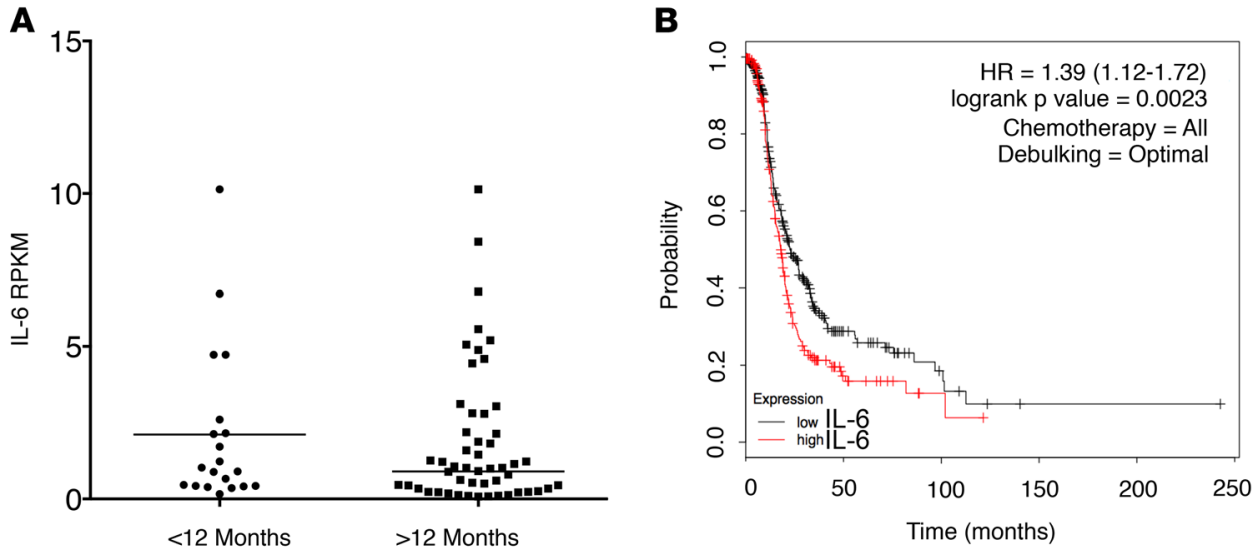

Figure 1. Increased IL-6 expression after chemotherapy associated with cancer progression and poor clinical outcome. (A) Mean of IL-6 expression (reads per kilobase of transcript, per million mapped reads; RPKM) in patients with free tumor survival duration less than 12 months and more than 12 months after initial chemotherapy. Data was drawn from TCGA ovarian cancer portal. There are 20 patients with free tumor survival duration less than 12 months and 22 patients with free tumor survival duration more than 12 months after initial chemotherapy. (B) Survival probability of patients with high-grade serous ovarian cancer correlates with IL-6 expression levels in tumors after chemotherapy $(P=0.0023)$.

$<0.001)$ compared with control (Figure 2E). In addition, increased $(P<0.05)$ expression of IL-6 and IL-6R in tumor residuals from carboplatin-treated mice vs. vehicle-treated animals was observed (Figure $2 \mathrm{~F}$ ). Taken together, these results demonstrated that platinum induces IL- 6 secretion by OC cells and further suggest that platinum-induced IL- 6 secretion contributes to enrichment of OCSC in residual tumors.

Upregulation of IL-6 signaling pathway regulates ALDH1A activity and OCSC formation. ALDH enzymatic activity is accepted as a marker of OCSC (4), and aldefluor activity of ALDH is determined primarily by ALDH1A1 isoform in $\operatorname{OCSC}(8,28)$. We determine the percentage of $\mathrm{ALDH}^{+}$cells and ALDH1A1 mRNA expression in a panel of OC cells. In whole-cell OC cultures, the percentage of $\mathrm{ALDH}^{+}$cells - determined by FACS analysis - ranged from $0.35 \%-45.6 \%$ (Supplemental Figure 1A), and ALDH1A1 expression varied from $0.1 \mathrm{pg} / \mathrm{ml}$ (OVCAR4) to $843.53 \mathrm{pg} / \mathrm{ml}$ (HeyA8) (Supplemental Figure 1B), with expression of ALDH1A1 and aldefluor activity being positively correlated in the majority of OCs (Supplemental Figure 1C) and suggesting that the ALDH1A1 is the main isoform contributing to aldefluor activity in OC cells.

To further investigate the functional role of IL- 6 signaling pathways in $\mathrm{ALDH}^{+}$OCSC, we examined IL-6 secretion and expression levels in OC-derived $\mathrm{ALDH}^{+/-}$cells. In Kuramochi cells OVCAR4 and A2780, expression of ALDH1A1 was greater $(P<0.01)$ in $\mathrm{ALDH}^{+}$compared with $\mathrm{ALDH}^{-}$cells (Supplemental Figure 2A). The level of IL- 6 secreted in the growth media by $\mathrm{ALDH}^{+}$cells was greater $(P<0.01)$ compared with respective $\mathrm{ALDH}^{-}$cells (Figure 3A), indicating that autocrine IL-6 secretion by $\mathrm{ALDH}^{+}$ cells correlated with OCSC phenotypes. Furthermore, expression of IL-6 and IL-6R expression was increased $(P<0.05)$ in $\mathrm{ALDH}^{+}$cells vs. respective $\mathrm{ALDH}^{-}$cells (Figure $\left.3 \mathrm{~B}\right)$. To further investigate the role of ALDH1A1 or IL-6 in OCSC, we knocked down ALDH1A1 or IL-6 expression in Kuramochi OC cells (Supplemental Figure 2B) and examined the effect on ALDH cell populations by FACS analysis. As shown in Figure 3C and Supplemental Figure 2B, ALDH1A1 knockdown cells decreased the percentage of $\mathrm{ALDH}^{+}$cells compared with the scrambled shRNA control cells (Kuramochi, shALDH1A1_1, $0.19 \% \pm 0.2 \%$ vs. shcontrol $2.8 \% \pm 0.2 \%$; shALDH1A1_2, $0.35 \% \pm 0.1 \%$ vs. shcontrol $2.8 \% \pm 0.2 \%$; OVCAR3, shALDH1A1_1, 5.84\% $\pm 2.78 \%$ vs. shcontrol $37.04 \% \pm 2.94 \%$; shALDH1A1_2, 3.67\% \pm $0.78 \%$ vs. shcontrol $37.04 \% \pm 2.94 \%$ ), confirming that expression of ALDH1A1 was positively related to ALDH activity. ALDH1A1 knockdown decreased IL-6R and IL-6 expression (Supplemental Figure 2C). Similar results were found in OVCAR3 OC cells with a relatively high baseline level of ALDH1A1 and IL-6 expression. ALDH1A1 knockdown in OVCAR3 decreased IL-6 expression, and IL-6 knockdown decreased ALDH1A1expression (Supplemental Figure 2D), further demonstrating that IL-6 and IL-6R expression correlated with ALDH1A1 expression in OC cells. Moreover, IL-6 knockdown reduced the percentage of $\mathrm{ALDH}^{+}$population ( Kuramochi, shIL-6_1, $0.79 \% \pm 0.2 \%$ vs. shcontrol $2.8 \% \pm 0.2 \%$; shIL-6_2, $0.28 \% \pm 0.1 \%$ vs. shcontrol $2.8 \% \pm 0.2 \%$; OVCAR3, shIL-6_1, 25.43\% $\pm 2.33 \%$ vs. shcontrol 

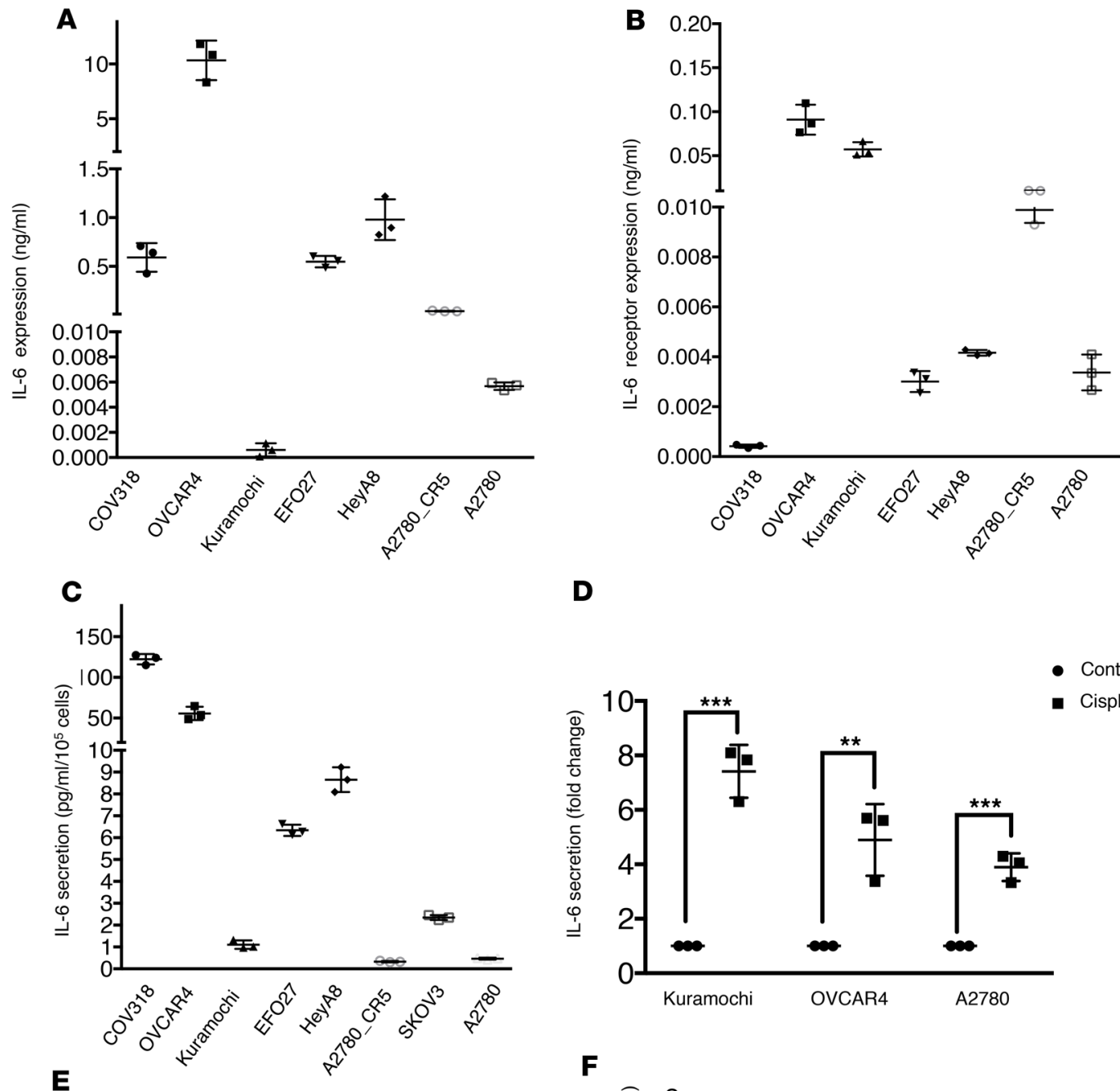

D
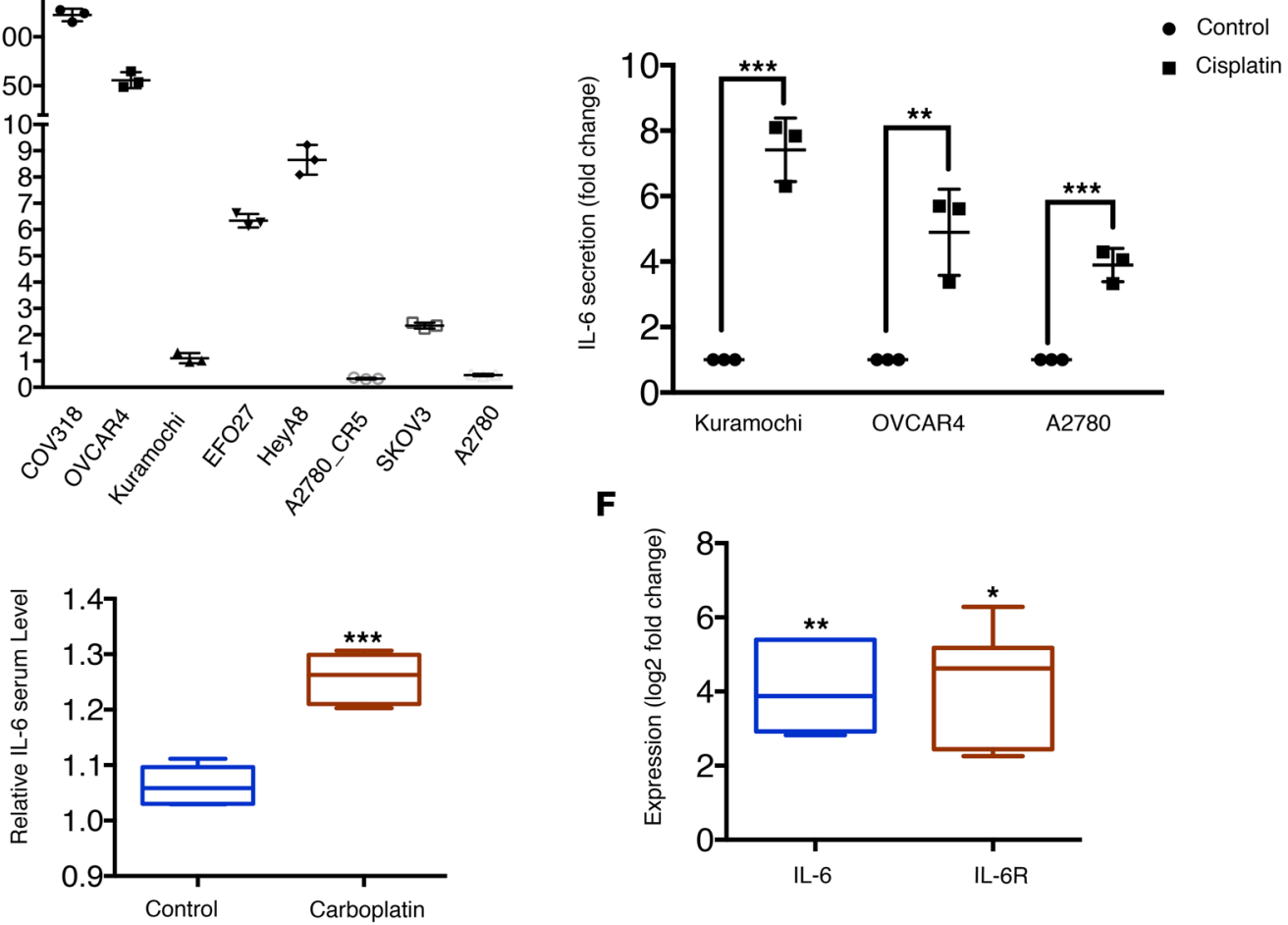

$\mathbf{F}$

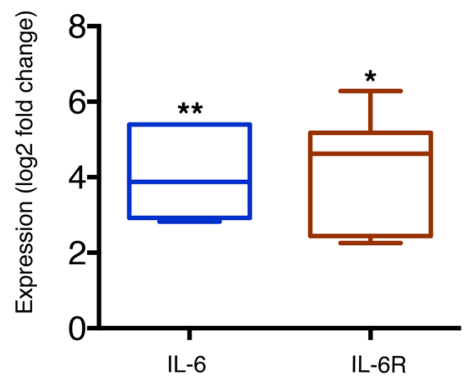

Figure 2. Platinum-induced IL-6 secretion contributes to enrichment of OCSC in residual tumors. (A) Basal expression of IL-6 and (B) IL-6 receptor mRNA expression in human OC cells $(\mathrm{ng} / \mathrm{ml})$ were measured by qPCR. Bars represent average measurements of 3 independent experiments \pm SD $(n=$ 3). (C) COV318, OVCAR4, Kuramochi, EFO-27, HeyA8, A2780_CR5, SKOV3, and A2780 OC cells were cultured under starving condition for 24 hours. ELISA was used to measure IL- 6 levels in the conditioned media. IL- 6 secretion $\left(\mathrm{pg} / \mathrm{ml} / 1 \times 10^{5}\right.$ cells) was determined and normalized to the cell number $(n=3)$.

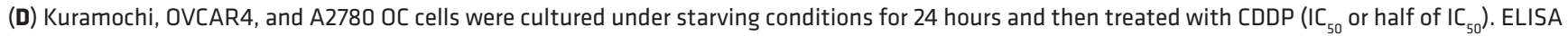
was used to measure IL-6 levels in the CM. IL-6 secretion ( $\mathrm{pg} / \mathrm{ml} / 1 \times 10^{5}$ cells) was determined and normalized to the cell number. Average fold change of IL-6 secretion ( \pm SD) of CDDP-treated cells compared with control-treated is shown (2-tailed Student's $t$ test, ${ }^{*} P<0.05,{ }^{* *} P<0.01$, and $\left.{ }^{* * *} P<0.001\right)(n$ = 3). (E) Nude mice were bearing A2780 i.p.-derived xenograft tumors were treated with carboplatin ( $50 \mathrm{mg} / \mathrm{kg}$, weekly for 3 weeks). Blood samples and xenograft tumors were collected after CDDP treatment. Average relative IL-6 expression level ( \pm SEM) in the plasma compared baseline plasma IL-6 level was measured by IL-6 ELISA and shown $(n=6)$. (F) IL-6 and IL-6R expression in the tumor residuals treated with control and carboplatin were measured by using qPCR. Mean fold change of IL-6 and IL-6R expression of 6 independent experiments is reported. Two-tailed Student's $t$ test was used to analyze statistical significance $\left({ }^{*} P<0.05,{ }^{* *} P<0.01\right.$, and $\left.{ }^{* * *} P<0.001\right)$. 
A IL-6 secretion in $\mathrm{ALDH}(+)$ vs. $\mathrm{ALDH}(-)$ cells

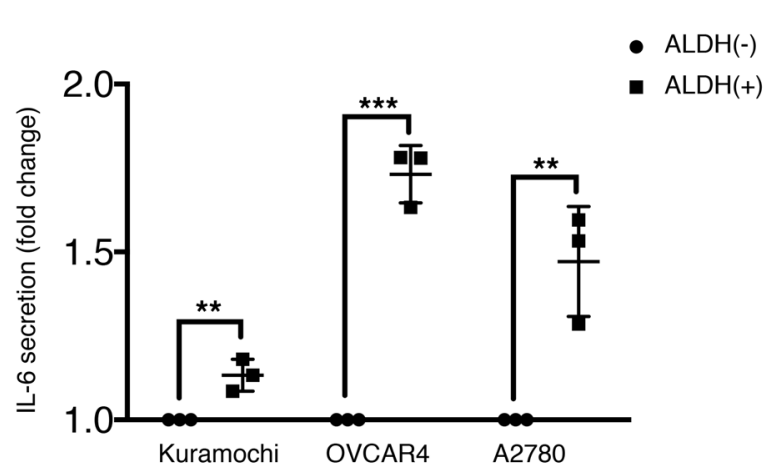

C

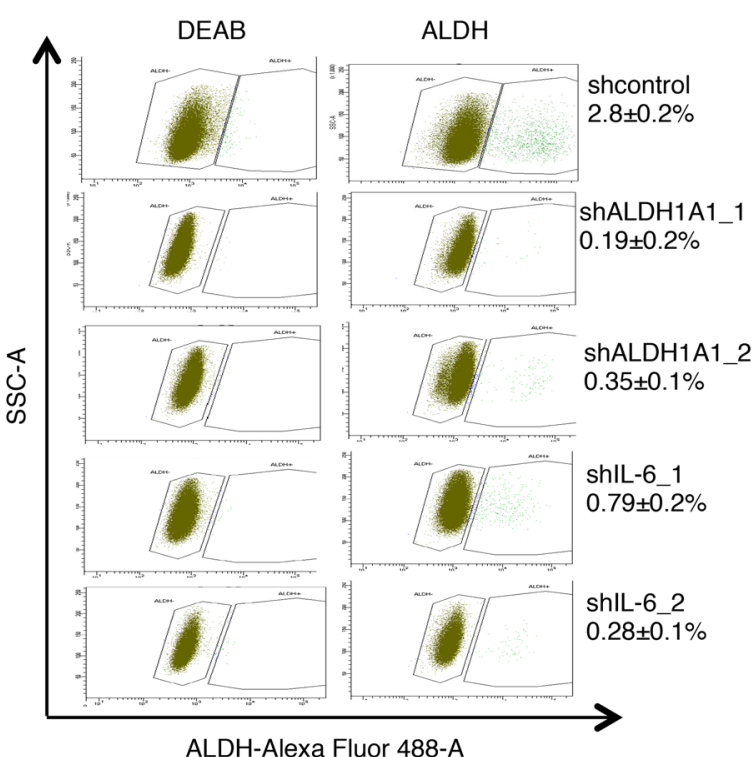

IL-6/L-6R expression in ALDH(+) vs. ALDH(-) cells

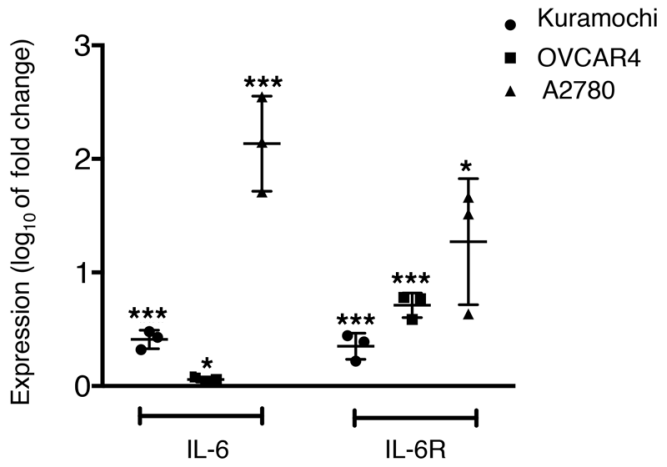

D

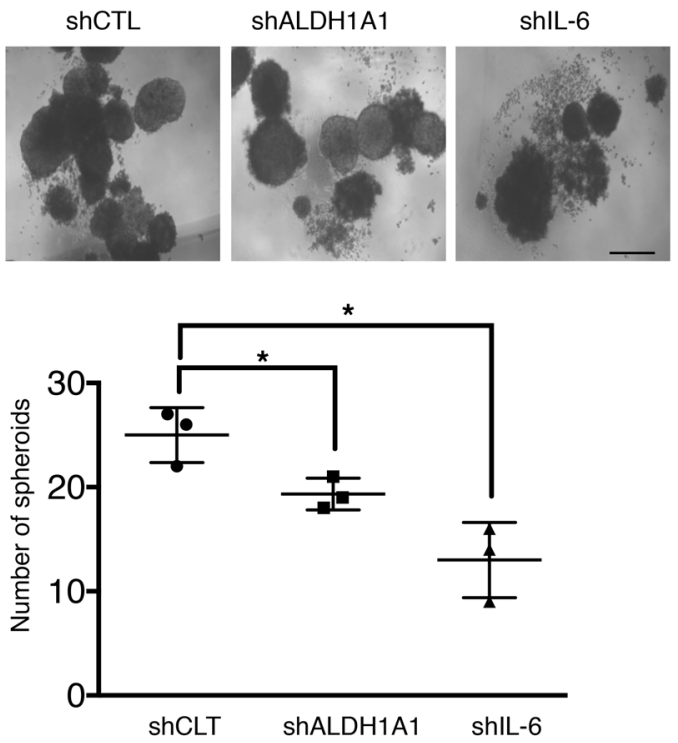

Figure 3. Upregulation of the IL-6 signaling pathway in ALDH+ ovarian cancer stem cells. (A) Five thousand $\mathrm{ALDH}^{+/}$cells were FACS sorted from Kuramochi, OVCAR4, and A2780 cells into 96-well plates. IL-6 levels were measured by IL-6 ELISA at 24 hours after starving conditions. Average relative IL- 6 secretion levels $( \pm \mathrm{SD})$ of $A L D H^{+}$OCs compared with $\mathrm{ALDH}^{-}$cells is shown $(n=3)$. (B) Expression of ALDH1A1, IL-6, and IL-6R were measured by qPCR in Kuramochi-, A2780-, and OVCAR4-derived ALDH+ cells, compared with respective $\mathrm{ALDH}^{-}$cells. Average fold change $( \pm \mathrm{SD})$ of 3 independent experiments is shown. $\left({ }^{*} P<0.05,{ }^{* *} P<0.01\right.$, and $\left.{ }^{* * *} P<0.001\right)(n=3)$. (C) Kuramochi cells were transfected with scrambled shRNA, shALDH1A1, or shIL-6 RNA. Side scatter of FACS analysis of the percentage of $\mathrm{ALDH}^{+}$cell population in Kuramochi cells with stable ALDH1A1 and IL-6 knockdown expression. Average percentage of $\mathrm{ALDH}^{+}$cells $( \pm \mathrm{SD})$ is shown below $(n=3$, 1 -way ANOVA, $\left.{ }^{*} P<0.05\right)$. (D) Representative pictures of spheroids formed by 500 shcontrol, shALDH1A1, and shIL-6 knockdown Kuramochi cells, which were cultured in stem cell culture condition for 14 days; the average number of spheroids $\left( \pm\right.$ SD) is shown below $\left(n=3,1\right.$-way ANOVA, $\left.{ }^{*} P<0.05\right)$. Scale bar: $100 \mu \mathrm{m}$.

$37.04 \% \pm 2.94 \%$; shIL-6_2, $16.70 \% \pm 4.66 \%$ vs. shcontrol 37.04\% $\pm 2.94 \%$ ) (Figure 3C and Supplemental Figure 2B) and ALDH1A1 protein expression (Supplemental Figure 2, C and D), supporting a regulatory role of IL-6 in ALDH1A1 expression in OC cells. In addition, spheroid formation was reduced $(P<0.05)$ in Kuramochi cells with stable knockdown of ALDH1A1 or IL-6 expression compared with scrambled shRNA ( $26 \pm 3$ vs. $19 \pm 2$ spheroids; $26 \pm 3$ vs. $13 \pm 3$ spheroids; Figure 3D), further supporting a role for ALDH1A1 and IL-6 in self-renewal and maintenance of OCSC. 
To further investigate downstream signals of IL-6 regulation of ALDH1A1 expression in OCSC, we examined STAT3 activation in $\mathrm{ALDH}^{+/-}$derived from OC cells. Compared with Kuramochi_ALDH ${ }^{-}$cells, the IL-6 signaling pathway was significantly upregulated in Kuramochi_ALDH ${ }^{+}$cells, indicated by overexpression of IL-6R, pSTAT3, and downstream target gene cyclin D1 (Supplemental Figure 2E). IL-6 knockdown in OC cells reduced expression of ALDH1A1 and STAT3 (Supplemental Figure 2, C and D). Collectively, these results suggest that the IL- 6 signaling pathway is upregulated in $\mathrm{ALDH}^{+} \mathrm{OC}_{\text {cells }}$ and plays a regulatory role in stem cell marker ALDH1A1 expression in OC cells.

Platinum and IL-6 induce ALDH1A1 and OCSC population. To determine whether IL-6 regulates ALDH1A1 transactivation, we used an ALDH1A1 promoter luciferase reporter assay. Treatment with IL- 6 transactivated the ALDH1A1 reporter gene expression in Kuramochi-derived $\mathrm{ALDH}^{-} \mathrm{OC}$ cells (Figure $4 \mathrm{~A}$ ), verifying the role of IL-6 in regulation of ALDH1A1 expression in OC cells. To further investigate the role of CDDP-induced IL-6 secretion in enrichment of $\mathrm{ALDH}^{+}$OCSC in OC, we examined aldefluor activity in OC cells treated with CDDP, IL-6, or IL-6-Nab. CDDP or IL-6 treatment alone increased the $\mathrm{ALDH}^{+}$population (Kuramochi, Figure 4B; A2780, Supplemental Figure 3A), and the CDDP-induced increase was abrogated by IL-6-Nab treatment (Figure 4B, Supplemental Figure 3A).

Next, to examine if CDDP- or IL-6-induced expression of ALDH1A1 in ALDH- OC cells results in conversion of aldefluor negative cells into aldefluor positive OCSC, Kuramochi $\mathrm{ALDH}^{-}$cells were treated with long-term CDDP or IL-6 (100 ng/ml, weekly for 3 weeks) and were analyzed by FACS. Either treatment increased $(P<0.01)$ the $\mathrm{ALDH}^{+}$population $(1.37 \% \pm 0.07 \%$ vs. $0.47 \% \pm 0.1 \%$, CDDP vs. control; $0.89 \% \pm 0.13 \%$ vs. $0.47 \% \pm 0.1 \%$, IL-6 vs. control) (Figure $4 \mathrm{C}$ ). To further verify the stemness features of CDDP- or IL-6-induced ALDH ${ }^{+}$OC cells, spheroid formation assays were performed. IL-6-treated (100 $\mathrm{ng} / \mathrm{ml}, 3$ weeks) A2780 $\mathrm{ALDH}^{-}$cells displayed enhanced $(P<0.05)$ self-renewal capability, as indicated by enhanced spheroid formation, compared with control-treated cells (Supplemental Figure 3B). After IL-6 treatment (72 hours), a dose-dependence increase in the $\mathrm{ALDH}^{+}$population in $\mathrm{ALDH}^{-}$cells was observed (Kuramochi, Supplemental Figure 3C; A2780, Supplemental Figure 3E), and increased $(P<0.05)$ expression of stemness-associated genes Sox2 and Bmil in the converted cells was observed (Kuramochi, Supplemental Figure 3D; A2780, Supplemental Figure 3F). In addition, A2780 CDDP-resistant OC cells (CR5) were more mesenchymal in appearance compared with parental A2780 (Supplemental Figure 4A), contained a greater percentage of $\mathrm{ALDH}^{+}$cells (Supplemental Figure 4B), and had higher mRNA expression of $A L D H 1 A 1, I L-6, I L-6 R$, Sox2, and Bmi1 than parental OC cells (Supplemental Figure 4C). Furthermore, Kuramochi cells with acquired resistance to CDDP (Supplemental Figure 4D) displayed increased $(P<$ 0.05 ) constitutive expression of ALDH1A1 (Supplemental Figure 4E), and CDDP treatment increased pSTAT3 and IL-6 protein levels (Supplemental Figure 4F), indicating that CDDP triggered activation of IL-6 signaling-mediated expression of ALDH1A1 in OC cells. In platinum-sensitive OC cells, IL-6 treatment activated STAT3, and the activation of pSTAT3 upon transient IL-6 exposure was maintained up to 12 hours (Supplemental Figure 4G). CDDP or IL-6 treatment increased $(P<0.05)$ mRNA expression of ALDH1A1, IL-6, and IL-6R (Supplemental Figure 4H) and ALDH1A1 protein level (Supplemental Figure $4 \mathrm{H}$ ) in platinum-sensitive OC cells — responses that were blocked by IL-6-Nab (Supplemental Figure 4, H and I). Taken together, these results indicate that CDDP-induced OCSC enrichment was partially due to IL-6 signaling pathway-mediated upregulation of ALDH1A1 expression in OCs.

To further investigate downstream signaling involved in IL-6-induced upregulation of ALDH1A1 expression in OCs, we used a pSTAT3 inhibitor (Stattic) to functionally inhibit constitutive activation of STAT3 in $\mathrm{ALDH}^{+}$OCSC. Treatment of Kuramochi-derived $\mathrm{ALDH}^{+}$cells with Stattic decreased $(P<0.05)$ the percentage of $\mathrm{ALDH}^{+}$cells (Figure 4D) and inhibited pSTAT3-induced expression of ALDH1A1 (Figure 4E), confirming that IL-6 signaling pathway mediated upregulation of ALDH1A1 expression in OCs via activation of STAT3.

IL-6-Nab-guadecitabine combination inhibits IL-6 signaling-mediated ALDH1A1 activation. The role of IL-6 in regulating DNA methytransferse-mediated gene silencing to promote tumorigenesis has been indicated in solid tumors $(24,25)$. We found that IL-6 induced upregulation of DNMT1 expression in Kuramochi-derived $\mathrm{ALDH}^{-}$cells (Supplemental Figure 5A) and, correspondingly, that IL-6 knockdown reduced DNMT1 expression (Supplemental Figure 5B). Together with our previous findings on DNMT1, $-3 A$, and $-3 B$ overexpression in $\mathrm{ALDH}^{+}$cells compared with $\mathrm{ALDH}^{-}$cells, and considering the fact that the DNMT inhibitor guadecitabine induced differentiation of $\mathrm{ALDH}^{+} \mathrm{OC}$ cells by upregulating differentiation genes HOXA10 and HOXA11 (4), we explored combining IL-6 blockade with DNMTi. To examine the effect of guadecitabine with IL-6-Nab on 

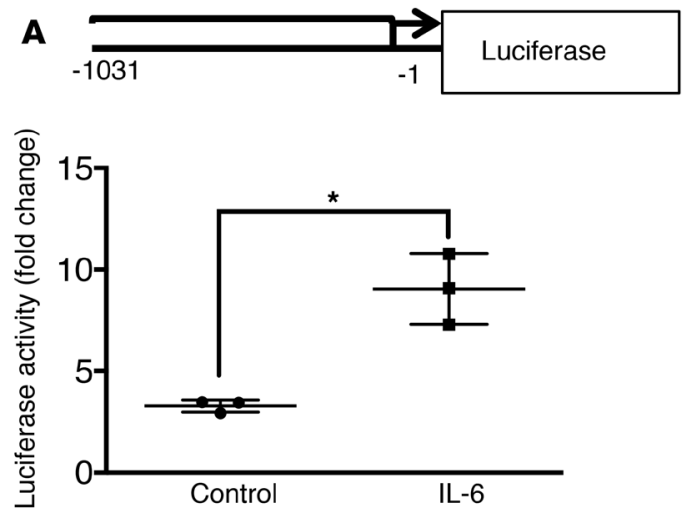

B

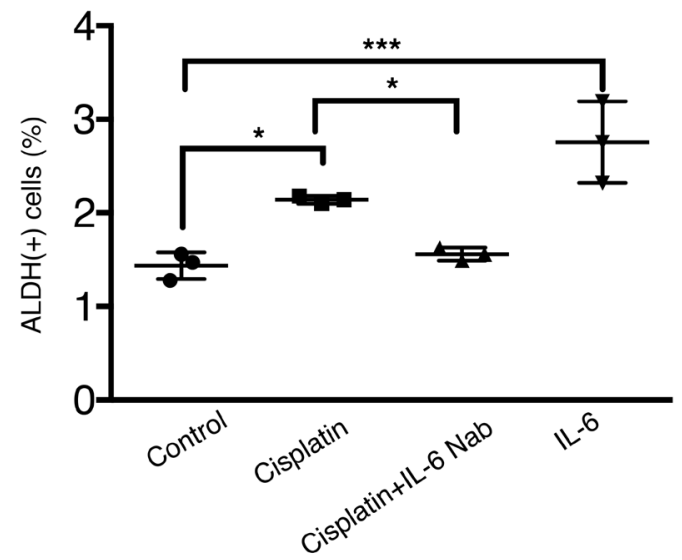

C

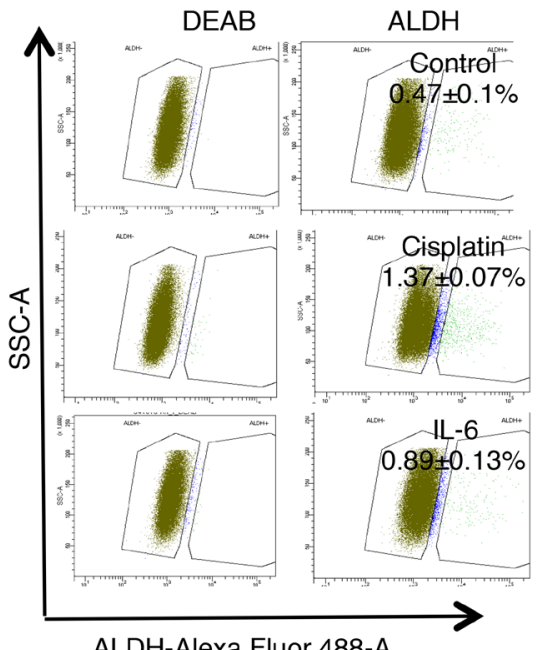

ALDH-Alexa Fluor 488-A

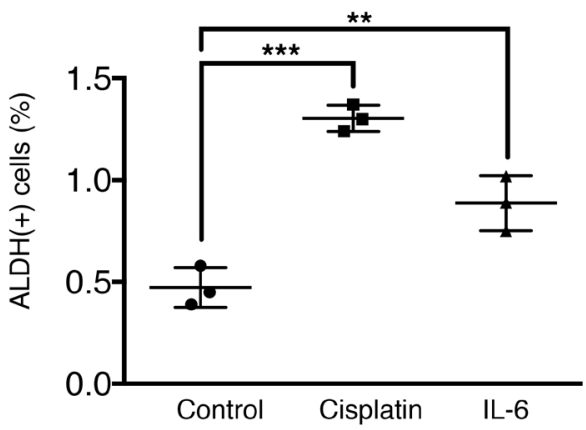

D

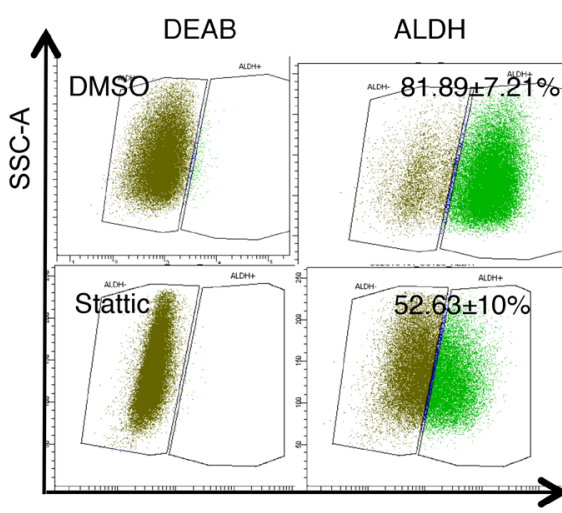

E

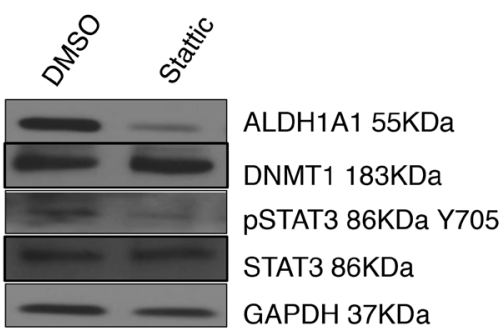

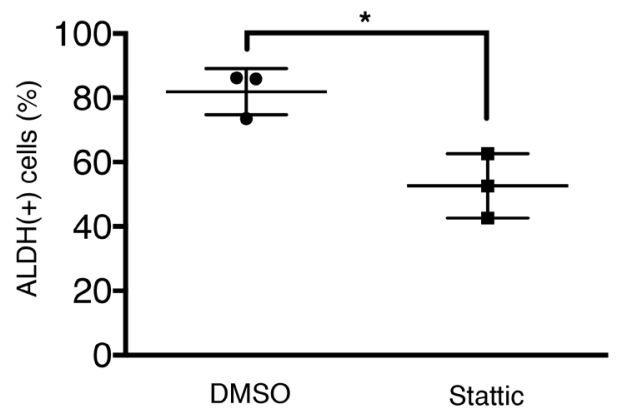

Figure 4. CDDP-induced IL-6 regulates ALDH1A expression and OCSC enrichment. (A) OC-derived (Kuramochi-derived) ALDH' cells were cotransfected with pGL3-ALDH1A1-Luc and renilla luciferase plasmid vector (pRL) or pGL3-Luc and pRL. Transfected cells were cultured with starving medium for 24 hours and were then treated with IL-6 $(100 \mathrm{ng} / \mathrm{ml})$. Luciferase signals recorded 3 hours after drug treatment. Renilla luciferase activity used for normalization. Average fold changes ( \pm SD) of relative luciferase unit (RLU) compared with pGL3 are shown $(n=3)$. (B) Quantification of FACS analysis of the percentage of ALDH in Kuramochi OC cells treated with CDDP ( $3 \mu \mathrm{M}, 3$ hours), CDDP $+\mathrm{IL}-6-\mathrm{Nab}(1 \mu \mathrm{g} / \mathrm{ml})$ and IL-6 $(500 \mathrm{ng} / \mathrm{ml})$ for 72 hours. Average percentage of ALDH ${ }^{+}$cells $( \pm$SD) is shown on the graph, and the quantification is shown ( $n=3,1$-way ANOVA, ${ }^{*} P<0.05$ and $\left.{ }^{* * *} P<0.001\right)$. (C) Side scatter of FACS analysis of percentage of $\mathrm{ALDH}^{+}$cells population in Kuramochi-derived $\mathrm{ALDH}^{-}$cells, which were treated with CDDP (IC ${ }_{50}$, weekly for 3 weeks) and IL-6 (100 ng/ml, weekly for 3 weeks). Average percentage of $\mathrm{ALDH}^{+}$cells $( \pm \mathrm{SD})$ is shown on the graph, and the quantification is shown $\left(n=3,1\right.$-way ANOVA, ${ }^{* *} P<0.01$ and $\left.{ }^{* * *} P<0.001\right)$. (D) FACS analysis of the percentage of Kuramochi-derived $\mathrm{ALDH}^{+}$cells treated daily with DMSO or Stattic $(3 \mu \mathrm{M})$ for 3 days. Average percentage of ALDH+ cells \pm SD is shown on the graph $(n=3)$. Two-tailed Student's $t$ test was used to analyze statistical significance $\left({ }^{*} P<0.05\right)$. (E) Protein expression of ALDH1A1, DNMT1, PSTAT3, STAT3, and GAPDH in DMSO or Stattic-treated Kuramochi_ALDH ${ }^{+}$cells were determined by Western blot $(n=2)$. 
OCSC, we treated Kuramochi_ALDH ${ }^{+}$cells with guadecitabine, IL-6-Nab alone, or the combination with the hypomethylating agent. Guadecitabine decreased $(P<0.01)$ the percentage of $\mathrm{ALDH}^{+}$cells (Figure $\left.5 \mathrm{~A}\right)$, and combining guadecitabine with IL-6-Nab further decreased $(P<0.05)$ the percentage of $\mathrm{ALDH}^{+}$cells (Figure 5A) and ALDH1A1 mRNA expression levels compared with either drug alone (Figure 5B). Similar results were observed in A2780 $\mathrm{ALDH}^{+}$cells (Supplemental Figure 5, C and D). To further investigate the molecular mechanism, $\mathrm{ALDH}^{+}$cells sorted from Kuramochi and A2780_CR5 were treated with guadecitabine, IL-6-Nab, or the combination. Guadecitabine partially decreased expression of ALDH1A1 and DNMT1, and IL-6-Nab alone reduced pSTAT3 expression but had little effect on ALDH1A1 expression. The IL-6-Nab- guadecitabine combination not only decreased endogenous expression of ALDH1A1, DNMT1, and pSTAT3 in ALDH ${ }^{+}$OCSC (Figure 5C, Supplemental Figure 5E), but also markedly blocked CDDP-induced upregulation of ALDH1A1, DNMT1, pSTAT3, and IL-6 expression in Kuramochi OC cells (Supplemental Figure 5F).

Combining IL-6-Nab with the hypomethylating agent guadecitabine inhibits survival of OCSC. Previous studies demonstrate that blocking IL- 6 enhances the antitumor and apoptotic activity of platinum chemotherapy in $\operatorname{OC}(29,30)$ and inhibiting ALDH1A1 induces apoptosis of OCSC $(4,31)$. To test the effect of combining IL-6-Nab with guadecitabine on CDDP chemosensitivity and stem cell properties, clonogenic survival, apoptosis assays, and spheroid formation were performed using OC cells treated with a single agent (IL-6$\mathrm{Nab}$, guadecitabine, CDDP) or in combination (IL-6-Nab + guadecitabine, CDDP + guadecitabine, CDDP + IL-6-Nab, CDDP + IL-6-Nab + guadecitabine). IL-6-Nab or guadecitabine increased $(P<0.0001)$ CDDP chemosensitivity of OC cells and decreased $(P<0.0001)$ the number of surviving colonies (Supplemental Figure 6A). Combining guadecitabine with IL-6-Nab augmented $(P<0.0001)$ the cytoxic effect of CDDP on OC clonogenic survival (Supplemental Figure 6A). To examine the effect of these combination treatments on self-renewal capability of OC cells, spheroid formation assays using ultra-low attachment conditions were performed on Kuramochi cells, followed by treatment with IL-6-Nab, guadecitabine alone, or in combination. Guadecitabine alone and with IL-6-Nab effectively reduced the numbers of spheroids formed by OC cells $(P<0.05)$ but not IL-6-Nab alone (Figure 5D).

Next, we examined the effect of guadecitabine and IL-6-Nab on $\mathrm{ALDH}^{+/-}$cells sorted from OC cells. Guadecitabine and IL-6-Nab had a greater effect on colony formation by $\mathrm{ALDH}^{+}$cells compared with treatment with guadecitabine alone (Figure 5E and Supplemental Figure 6B), although guadecitabine inhibited $(P<0.05)$ clonogenicity $\mathrm{ALDH}^{+}$compared with control treatment (Figure 5E and Supplemental Figure 6B). Guadecitabine alone or with IL-6-Nab resensitized $\mathrm{ALDH}^{+}$cells to CDDP treatment $(P<$ 0.001), and the effect of the combination therapy on resensitization of $\mathrm{ALDH}^{+}$cells to CDDP treatment was greater than either agent alone (Figure 5E and Supplemental Figure 6B). Guadecitabine or IL-6-Nab increased apoptosis in $\mathrm{ALDH}^{+}$cells, indicated by caspase 3/7 cleavage activity (Supplemental Figure 6 , $\mathrm{C}$ and $\mathrm{D})$; however, greater $(P<0.001)$ cell death was induced by the combination of guadecitabine with IL-6-Nab (Supplemental Figure 6, C and D). By inhibiting expression of ALDH1A1 and blocking IL-6/ IL-6R/STAT3 signaling pathway activation, the IL-6-Nab-guadecitabine combination reduced OC cell survival, increased OC cell chemosensitivity, and induced apoptosis.

Combining IL-6-Nab with guadecitabine reduces platinum-induced enrichment of OCSC in vivo and prevents tumor relapse. To investigate the efficacy of IL-6-Nab plus guadecitabine as a potential postplatinum maintenance strategy that prevents enrichment of the $\mathrm{ALDH}^{+}$stem cell population in vivo, we used an i.p. xenograft model derived from OC cells (A2780). Nude mice bearing i.p. tumors were treated with carboplatin (50 $\mathrm{mg} / \mathrm{ml}$, weekly) for 3 weeks and were then randomized to a 2-week, twice-weekly treatment with vehicle, IL-6-Nab, or guadecitabine alone or in combination (Figure 6A). Based on body weight measurements, single and combination treatments (chemotherapy followed by maintenance treatment) were well tolerated (Supplemental Figure 7A). Treatment with carboplatin effectively decreased $(P<0.001)$ total i.p. tumor weight and the number of metastases (Figure 6, B and C, and Supplemental Figure 7, B and C). However, tumor relapse was observed 2 weeks after 3-week carboplatin treatment (Figure 6, B and C). Although guadecitabine treatment alone decreased $(P<0.05)$ recurrent tumor weight compared with vehicle-treated mice, the combination of IL-6-Nab-guadecitabine was more effective $(P<0.05)$ at reducing platinum-treated recurrent tumor weight and metastases (Figure 6, B and C) and at preventing $(P<0.05)$ tumor relapse (Supplemental Table 2). IL-6-Nab alone had no effect on recurrent tumor growth (Figure 6B).

Xenograft tumors were collected and dissociated to single cell suspension at indicated time points (Figure 6A), and cells were analyzed for $\mathrm{ALDH}^{+} \mathrm{OCSC}$ population by FACS and for self-renewal ability by the spheroid formation assay. Following carboplatin treatment, percentage of $\mathrm{ALDH}^{+}$cells was increased 
A
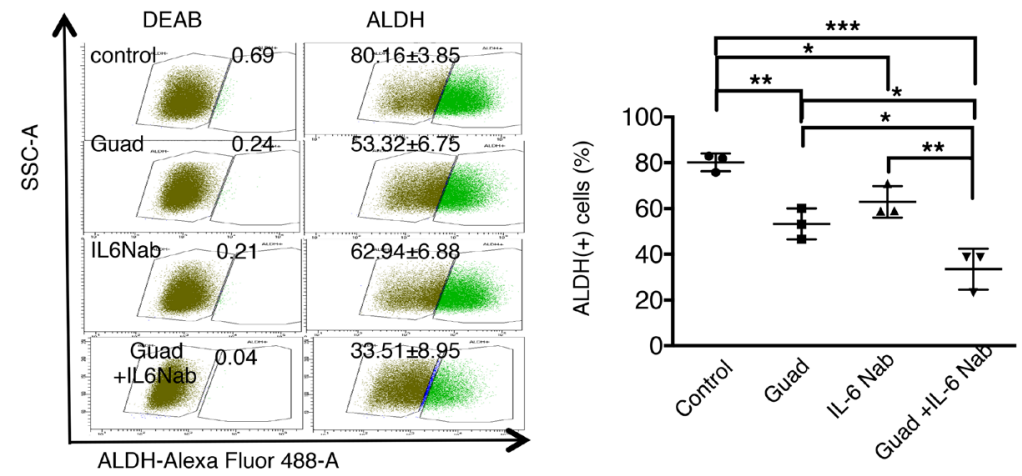

\section{B}

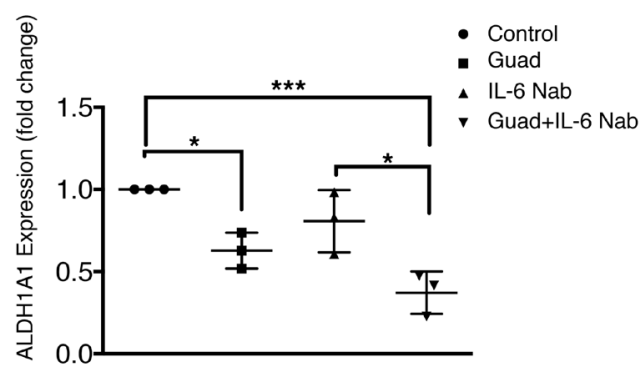

C

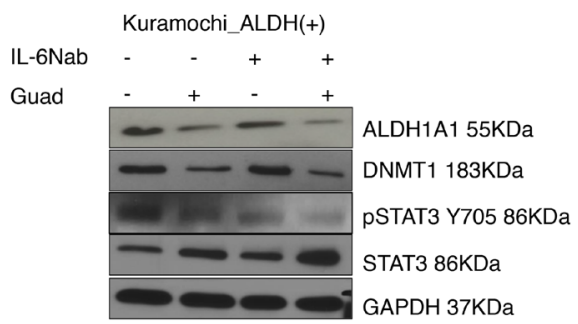

D

Control

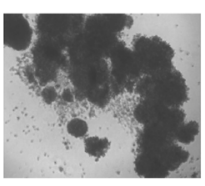

Guad

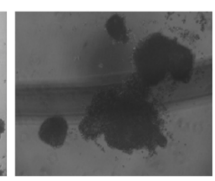

IL-6Nab

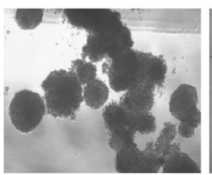

Guad+IL-6Nab
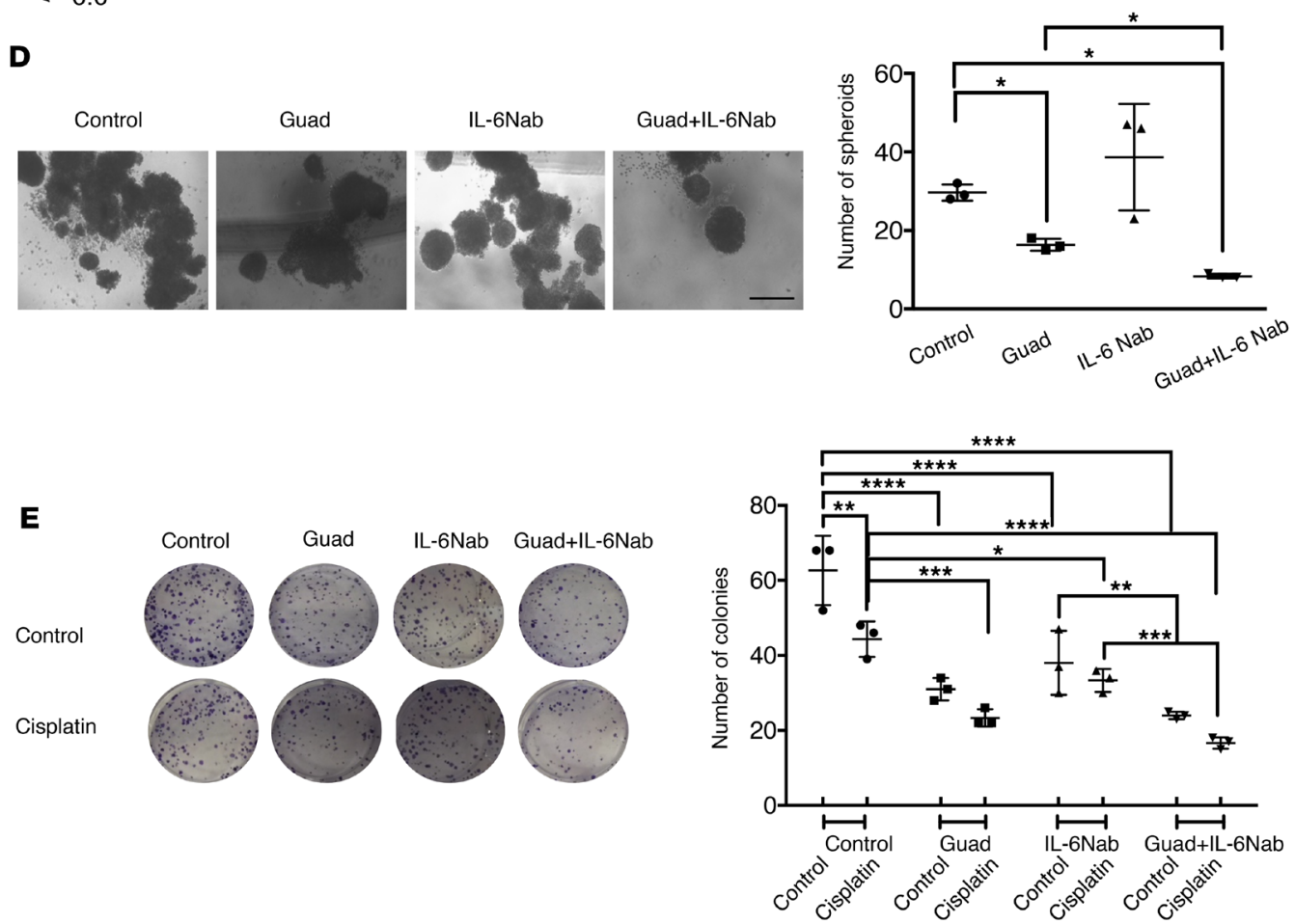

Figure 5. IL-6-Nab-guadecitabine combination eliminates ALDH+ cells by inhibiting ALDH1A1 expression. (A) FACS analysis of the percentage of Kuramochi-derived $\mathrm{ALDH}^{+}$cells treated daily with guadecitabine $(100 \mathrm{nM}), \mathrm{IL}-6-\mathrm{Nab}(1 \mu \mathrm{g} / \mathrm{ml})$, or IL-6-Nab- guadecitabine for 4 days. Average percentage of ALDH ${ }^{+}$ cells $\pm S D$ is shown on the side scatter graph (left), and the quantification is shown (right) ( $n=3,1$-way ANOVA). (B) Average fold change of ALDH1A1 expression $\pm \mathrm{SD}$ in Kuramochi-derived $\mathrm{ALDH}^{+}$cells treated with guadecitabine, IL-6-Nab, or IL-6-Nab-guadecitabine compared with control cells was determined by qPCR $\left(n=3\right.$, 1-way ANOVA, ${ }^{*} P<0.05$ and $\left.{ }^{* * *} P<0.001\right)$ (C) Kuramochi-derived ALDH ${ }^{*}$ cells were treated daily with guadecitabine (100 $\left.\mathrm{nM}\right)$, IL-6$\mathrm{Nab}(1 \mu \mathrm{g} / \mathrm{ml})$, or IL-6-Nab-guadecitabine for 4 days. The protein expression of ALDH1A1, DNMT1, pSTAT3, STAT3, and GAPDH was determined by western blot $(n=2)$. (D) Spheroid formation assay of 500 conditioned Kuramochi cells, which were treated with control, IL-6-Nab (400 ng/ml), and guadecitabine (100 nM, 3 days) or in combination. Cells were directly sorted in 96 low-attached plates and cultured in stem cell condition for 14 days. Representative images and the average number of spheroids \pm SD are shown in the graph. Scale bar: $100 \mu m\left(n=3,1\right.$-way ANOVA, $\left.{ }^{*} P<0.05\right)$. (E) Kuramochi_ALDH ${ }^{+}$cells were treated daily with guadecitabine $(100 \mathrm{nM})$, IL-6-Nab $(1 \mu \mathrm{g} / \mathrm{ml})$, or IL-6-Nab- guadecitabine for 4 days. Five hundred pretreated cells were reseeded into 6 -well plates for clonogenic survival assays. Representative images and the average number and of colonies formed by pretreated Kuramochi_ALDH ${ }^{+}$ cells with the conditions described previously and pretreated Kuramochi_ALDH ${ }^{+}$cells after exposure to cisplatin ( $\left.3 \mu \mathrm{M}\right)$ for 3 hours ( $n=3,2$-way ANOVA, Dunnett's and Sidak's multiple comparisons tests used for multiple comparison, ${ }^{*} P<0.05,{ }^{* *} P<0.01,{ }^{* * *} P<0.001$, and ${ }^{* * * *} P<0.0001$ ). 
$(P<0.001)$ in tumor residuals (carbo-residuals and carbo-recurrent) compared with control (Figure 6D and Supplemental Figure 8A). Maintenance treatment with guadecitabine alone or IL-6-Nab alone reduced ( $P$ $<0.001)$ the percentage of $\mathrm{ALDH}^{+}$cells in the tumor residuals, which was further decreased $(P<0.001)$ by IL-6-Nab-guadecitabine compared with either treatment alone (Figure 6D and Supplemental Figure 8A). OC cells isolated from carbo-residual and carbo-recurrent tumors demonstrated an enhanced $(P<0.001)$ ability to form spheroids compared with controls (Figure 6E and Supplemental Figure 8B), which was inhibited $(P<0.001)$ by maintenance treatment with guadecitabine or IL-6-Nab alone and further reduced by the combination therapy (Figure $6 \mathrm{E}$ and Supplemental Figure $8 \mathrm{~B}$ ).

In carboplatin-treated tumor residuals and recurrent tumors, increased $(P<0.05)$ expression of $I L-6$, $I L-6 R, A L D H 1 A 1$, Sox2, and Bmi1 were observed (Figure 6F), supporting the possibility that platinum induces IL-6 signaling pathway-mediated ALDH1A1 expression in OC cells, resulting in enrichment of $\mathrm{ALDH}^{+} \mathrm{OCSC}$. Single-agent guadecitabine treatment decreased $(P<0.01)$ expression of stemness genes $(A L D H 1 A 1$ and Bmi1) and DNMT1 protein levels in recurrent tumors (Figure 6F and Supplemental Figure 8C). Maintenance treatment with IL-6-Nab decreased $(P<0.01)$ expression of $I L-6, I L-6 R, A L D$ $H 1 A 1$, Sox2, and Bmi1 (Figure 6F) and reduced protein levels of ALDH1A1 and cyclin D1 (Supplemental Figure 8C). The negligible effect of IL-6-Nab on STAT3 activation in recurrent tumors compared with vehicle-treated recurrent tumors was likely due to the short signal duration of pSTAT3. However, downregulation of cyclin D1 in IL-6-Nab-treated recurrent tumors further indicated the overall inhibition of the IL-6/IL-6R/STAT3 signaling pathway in IL-6-Nab-treated xenografts (Supplemental Figure 8C). The IL-6-Nab-guadecitabine combination (2 weeks of treatment followed by 3-week carboplatin chemotherapy) markedly inhibited $(P<0.001) A L D H 1 A 1$, Sox2, Bmi1, IL-6, and $I L-6 R$ expression, along with DNMT1, ALDH1A1, pSTAT3, and CyclinD1 in recurrent tumors compared with vehicle treated tumors (Figure 6F and Supplemental Figure 8C). Carboplatin treatment increased $(P<0.05)$ human IL-6 levels in the circulation, which was reduced $(P<0.05)$ by either IL-6-Nab alone or IL-6-Nab-guadecitabine (Supplemental Figure 8D), suggesting that the anti-OCSC effect of guadecitabine with IL-6-Nab was partially due to inhibiting IL-6 signaling pathways and DNMT1 expression.

Role of the microenvironment in IL-6 secretion. The tumor-associated stroma including endothelial cells, fibroblasts, and adipocytes $(23,32,33)$ serves as a reservoir for paracrine inflammatory factors, such as IL-6, contributing to the formation of a favorable niche for CSC and maintenance and expansion of the OCSC population (23). To investigate the role of the microenvironment in CDDP-induced IL-6 secretion, baseline IL-6 secretion level in normal omental fibroblasts (NOFs) growth media was examined. Basal IL-6 secretion by NOFs was significantly $(P<0.05)$ higher than OC cells $(418.4 \pm$ $1.4 \mathrm{pg} / \mathrm{ml} / 1 \times 10^{5} \mathrm{NOF}$ vs. A2780, $0.48 \pm 0.01 \mathrm{pg} / \mathrm{ml} / 1 \times 10^{5}$ cells; Kuramochi, $1.35 \pm 0.04 \mathrm{pg} / \mathrm{ml} /$ $1 \times 10^{5}$ cells; OVCAR4, $51.3 \pm 3.4 \mathrm{pg} / \mathrm{ml} / 1 \times 10^{5} \mathrm{cells}$ ) (Figure 7A). Furthermore, after CDDP treatment, IL-6 secretion by NOFs was increased compared with nontreated cells (Figure 7B), indicating a potential role of paracrine IL-6 from NOFs in contributing to OCSC enrichment. Moreover, CDDP induced IL-6 secretion by coculturing OC cells with NOFs (Figure 7B), suggesting the role of NOFs in CDDP-induced IL-6 secretion. Finally, to further examine whether the microenvironment plays a role in cytokine IL-6 regulation of ALDH1A1 expression in OCs, we cocultured OC-derived ALDH cells (Kuramochi and A2780) with NOFs in a transwell cocultured system (Figure 7C). Coculture with NOFs increased endogenous mRNA expression of ALDH1A1, Sox2, and Bmi1 in OC ALDH ${ }^{-}$cells compared with respective $\mathrm{ALDH}^{-}$cells cultured alone (Figure 7, D and E).

\section{Discussion}

It has been suggested that cellular plasticity allows nontumorigenic cancer cells, under environmental stimuli, to dedifferentiate and enter the CSC pool, contributing to therapy resistance and disease progression. Here, we demonstrate in mouse models replicating treatment and the clinical course of OC that platinum-induced activation of IL-6 signaling promotes enrichment in OCSC. We provide preclinical evidence for a potentially novel anti-IL6 + epigenetic intervention to eradicate OSCS in residual tumors. Our data have important consequences.

First, we show that both IL-6 and its receptor are highly expressed in $\mathrm{ALDH}^{+}$OCSC compared with $\mathrm{ALDH}^{-}$non-OCSC, indicating the potential role of IL-6 in regulation of OCSC. In addition, we report that chemotherapy-linked inflammation, which was previously detected in clinical samples (14, 34 ), is associated with upregulation of IL-6, which in turn is associated with poor clinical outcome and low survival rate. Emerging evidence points to enrichment of the OCSC population in residual tumors 


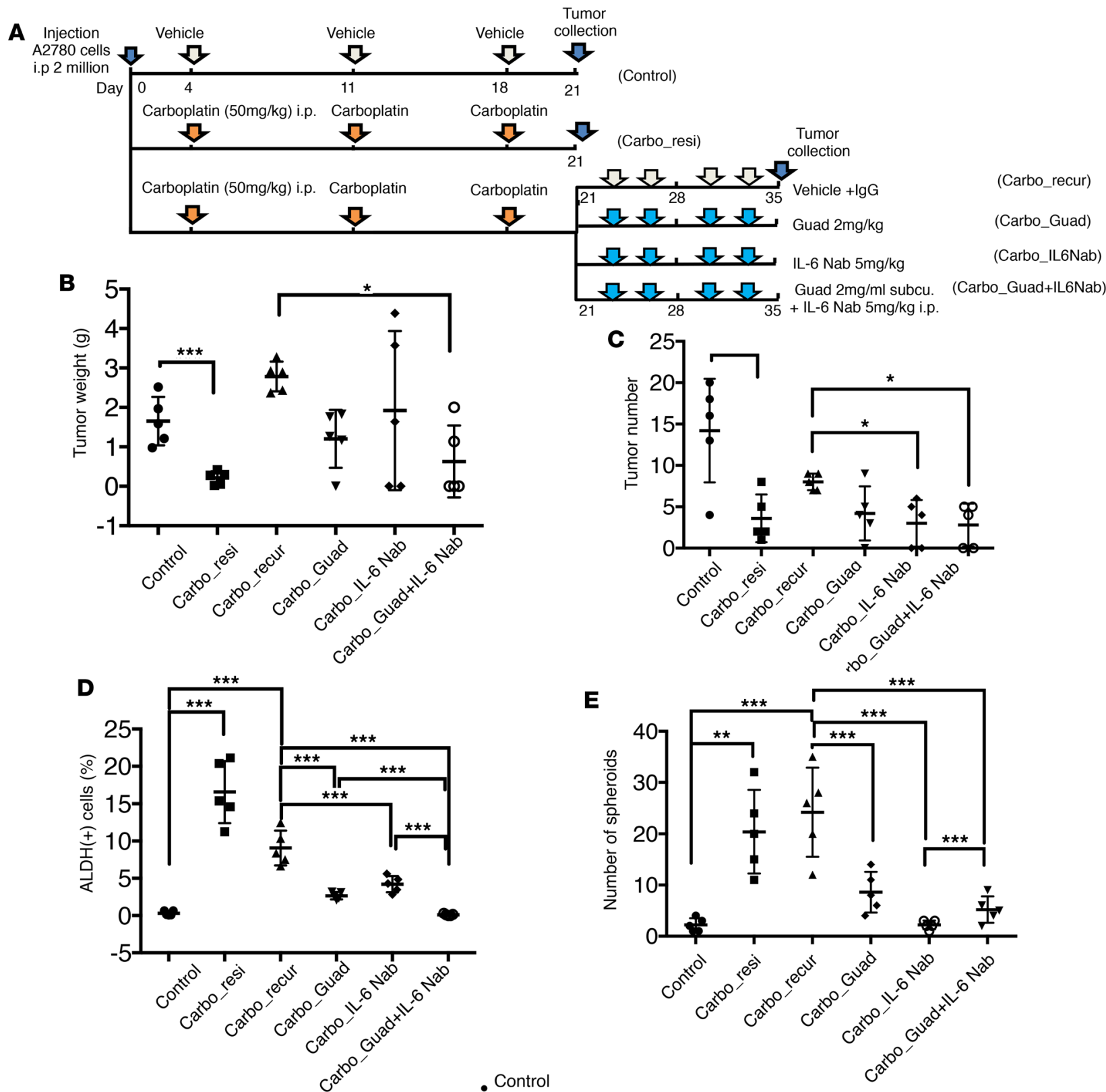

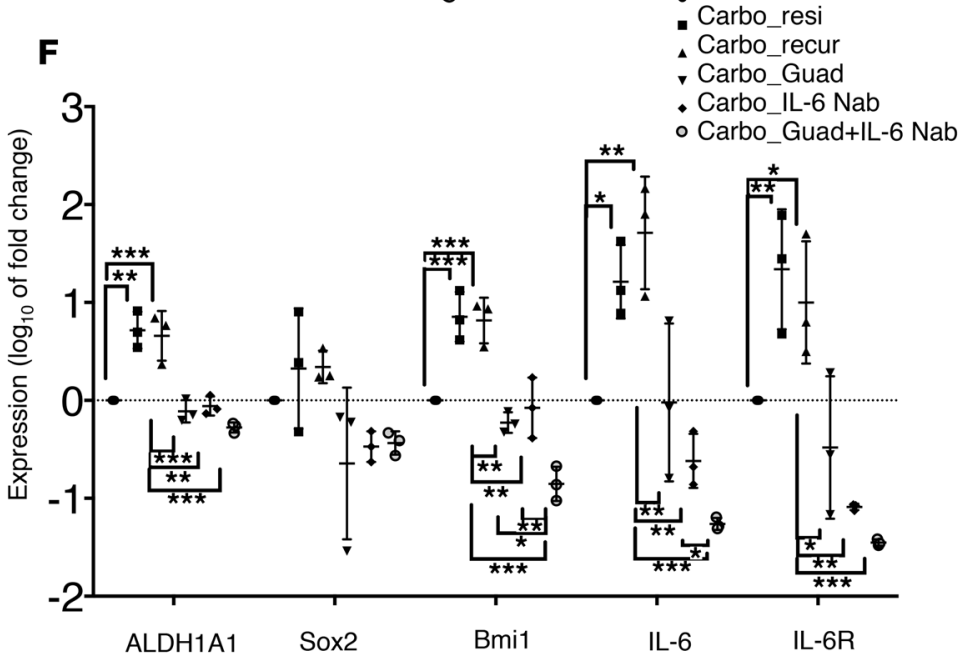

Figure 6. Combination of IL-6 neutralizing antibody with guadecitabine as maintenance therapy reduces $\mathrm{ALDH}^{+}$population in platinum-treated tumor residuals. (A) Schematic diagram of experimental design including carboplatin treatment phase followed by randomization to vehicle, guadecitabine, IL-6-Nab or the drug combination. (B-D) Effects of different treatment strategies on total tumor weight $\left(n=5,1\right.$-way ANOVA, ${ }^{*} P<$ 0.05 and $\left.{ }^{* *} P<0.001\right)(B)$, total number of tumor metastatic sites ( $n=5,1$-way ANOVA, $\left.{ }^{*} P<0.05\right)(\mathbf{C})$, and average of the percentage of $\mathrm{ALDH}^{+}$cells in xenografts $(n=5,1$-way ANOVA, ${ }^{* * *} P<0.001$ ) (D). (E) The average number $\pm \mathrm{SD}$ of spheroids formed by 10,000 cells dissociated from xenografts, which were treated with vehicle, carboplatin, carboplatin + vehicle (Carbo_resi), carboplatin + guadecitabine, carboplatin + IL-6-Nab, or carboplatin + guadecitabine + IL-6-Nab ( $n=5$, 1-way ANOVA, ${ }^{* *} P<0.01$ and ${ }^{* *} P<0.001$ ). (F) mRNA expression of ALDH1A1, Sox2, Bmi1, IL-6, and IL-6 receptor ( $n=3,1$-way ANOVA, ${ }^{*} P<$ $0.05,{ }^{* *} P<0.01$, and ${ }^{* *} P<0.001$ ) 
A Baseline IL-6 secretion in NOFs and OCs

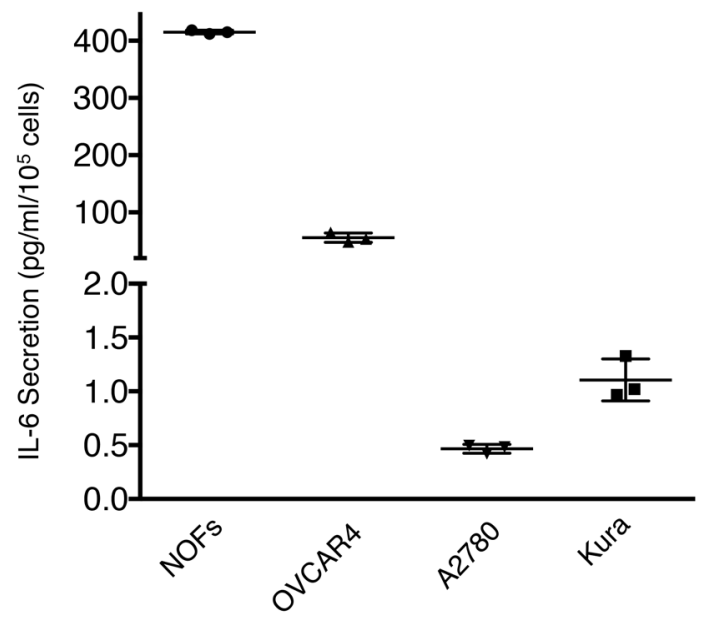

B Cisplatin-induced IL-6 secretion in NOFs/+OCs
C

2D Culture

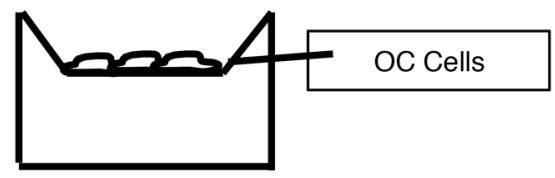

Transwell Co-Culture

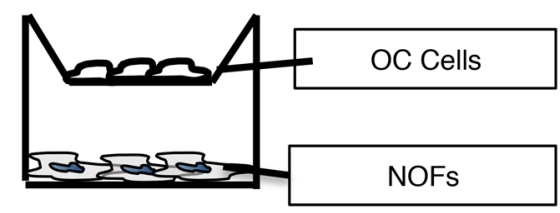

E

A2780_ALDH(-)+NOFs vs. ALDH(-)

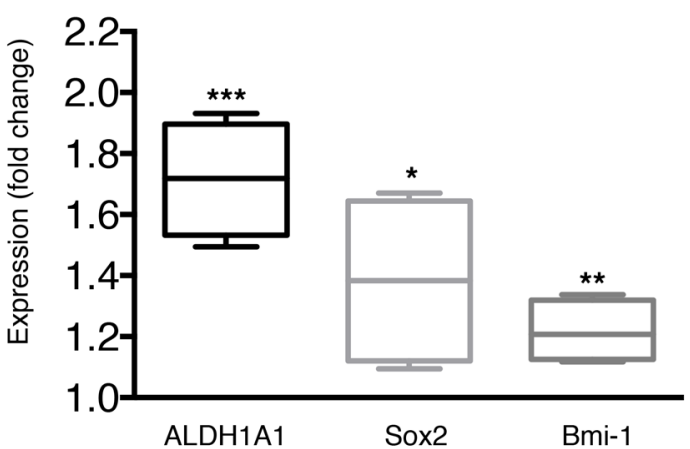

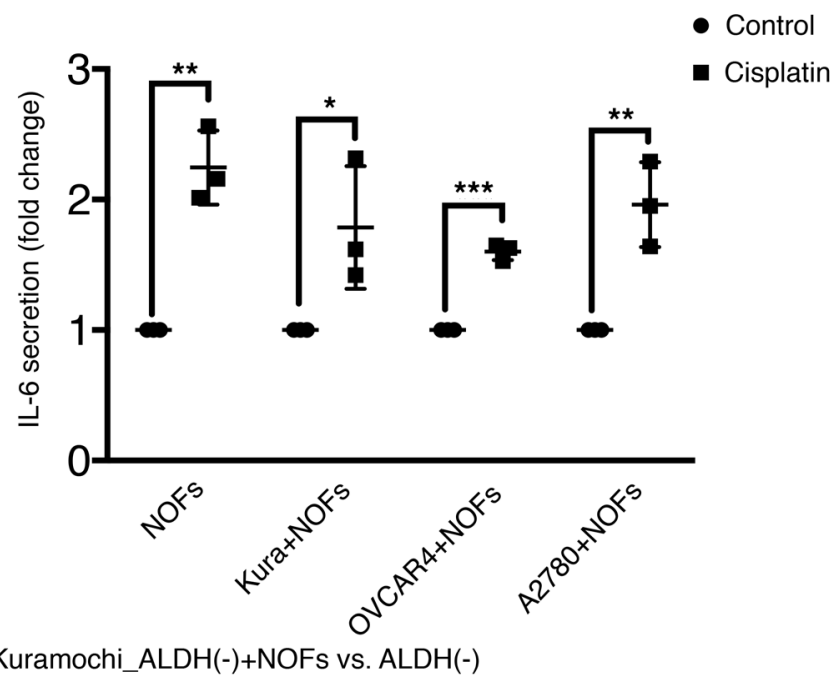

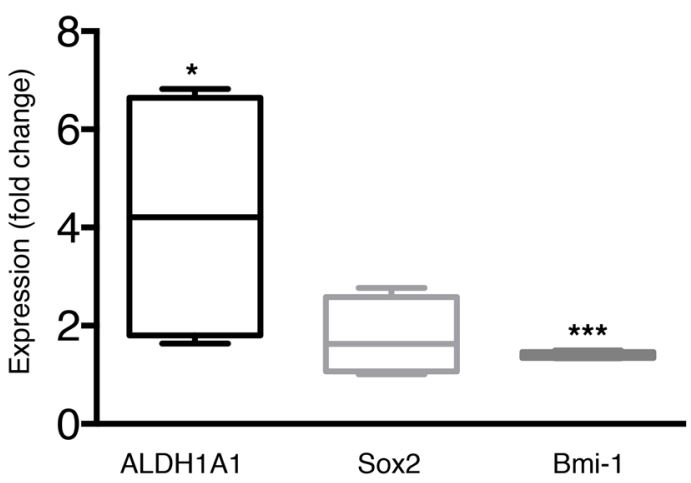

Figure 7. Normal omental fibroblasts (NOFs) enhance IL-6 secretion. (A) Baseline IL-6 secretion level by Kuramochi, OVCAR4, A2780, and NOFs $(n=3)$. (B) NOFs cells were cultured alone or cocultured with OCs (Kuramochi + NOFs, OVCAR4 + NOFs, $A 2780+N O F s)$ under starving condition for 24 hours and then treated with CDDP (IC 50 or half of $\left.\mathrm{IC}_{50}\right)$. IL- 6 levels in the conditioned media were measured at 24 hours after exposure to CDDP by ELISA. IL- 6 secretion ( $\mathrm{pg} / \mathrm{ml} / 1 \times 10^{5}$ cells) was determined and normalized to the cell number. Average relative IL-6 secretion ( \pm SD) of CDDP-treated cells compared with control-treated is shown in the graph ( $n=3$, 2-tailed Student's $t$ test, ${ }^{*} P<0.05$, ${ }^{* *} P<0.01$, and $\left.{ }^{* *} P<0.001\right)$. (C) Schematic diagram of transwell coculture system. In this $0.45-\mu \mathrm{m}$ transwell coculture system, OC cells (Kuramochi_ALDH ${ }^{-} / A 2780 \_A L D H^{-}$cells) with starving medium (DMEM supplemented with $0.5 \%$ FBS) were seeded in the upper chambers alone (top) or with NOFs, which were seeded on the bottom chamber in DMEM supplemented with $0.5 \%$ FBS (bottom). (D and $\mathbf{E})$ Average fold change with range of mRNA ALDH1A1, Sox2, and Bmi1 expression in (D) Kuramochi- and (E) A2780-derived ALDH' cells cocultured with NOFs compared with respective $\mathrm{ALDH}^{-}$cells alone shown in the graph (average fold change \pm SD). Twotailed Student's $t$ test was used to analyze statistical significance $\left(n=3,{ }^{*} P<0.05\right.$, ${ }^{* *} P<0.01$, and $\left.{ }^{* * *} P<0.001\right)$.

after platinum therapy $(4,35)$. We and others have shown that increased IL-6 secretion in OC cells after platinum treatment contributes to chemoresistant OC $(18,30)$, and here we show a direct connection between platinum-induced activation of the IL- 6 signaling pathway and OCSC enrichment. The molecular mechanism for IL-6 upregulation in OC cells upon cisplatin treatment is partially due to activation of noncoding RNA HOTAIR, which has been demonstrated in our previous study. Plat- 
inum-induced DNA damage results in upregulation of HOTAIR expression, which in turn results in sustained activation of DNA damage response after platinum treatment (36). We further showed that platinum-induced expression of HOTAIR activated NF- $\mathrm{kB}$ activation during DNA damage response and upregulated downstream target gene IL-6 expression (36).

Second, we demonstrate that treatment-induced changes in stromal cells drive non-CSCs to CSC conversion. The TME serves as a reservoir for inflammatory factors contributing to the formation of a CSC favorable niche (23) and also plays an important role in regulating epigenetic events associated with CSC maintenance in tumors (37). Within the tumor sites, reciprocal crosstalk between immune, stromal, and cancer cells plays a crucial role in deregulating epigenetic-mediated gene silencing and driving tumor initiation and tumor progression (19-22). CAFs in particular $(20,22)$ are involved in inducing genome-wide methylation alternation required for epithelial-to-mesenchymal transition (EMT) and maintenance of the stemness in prostate cancer cells (22). Along with fibroblasts, immune components - including myeloid and T cells, which secrete chemokines CXCL12, CXCL20 and CXCL8 - play key roles in maintaining the CSC niche $(38,39)$. It has been shown that coculture of NOFs with OC cells converts NOFs into CAFs (17), and here we demonstrate a role for NOFs in platinum-induced IL-6 secretion and promoting enrichment of OCSC. Furthermore, recent studies highlight the epigenetic effects of chronic inflammation and immune cells that promote tumorigenesis, including IL-6, which has been shown to upregulate DNMT1, leading to DNMT-mediated gene silencing and tumorigenesis in gastric and colon cancers $(19,24,25)$. Here, we further demonstrate that IL-6 induced DNMT1 expression in OC cells, supporting the rationale to combine IL-6 signaling blockade with HMA to eliminate OCSC and prevents recurrent OC.

An emerging model based on recent findings that hierarchically organized cancer cell populations are more plastic than previously appreciated $(35,40)$ supports our findings that cancer cells may dedifferentiate and reenter the CSC pool under the influence of extrinsic factors. Although IL- 6 has been identified as an important regulator of self-renewal of CSCs in other tumor types $(13,32)$, the precise molecular mechanisms by which IL-6 regulate CSC in OC has not been clearly defined. Here, we demonstrate autocrine and paracrine roles for IL-6 in regulation of ALDH1A1 expression in OC cells. Expression of IL-6 and/or IL-6R expression correlates with ALDH1A1 expression, and aberrant activation of IL-6 signaling pathways in OC cells contributes the enrichment of OCSC in tumors after platinum treatment. Further, the IL-6/STAT3 signaling pathway is more activated in $\mathrm{ALDH}^{+} \mathrm{OC}$ cells compared with $\mathrm{ALDH}^{-} \mathrm{OC}$ cells, and STAT3 phosphorylation is strongly associated with the expression of ALDH1A1. Similar findings have been reported in colon (41) and breast (42) CSCs, indicating the role of IL-6/STAT3 in maintenance of ALDH ${ }^{+}$CSCs and conversion of non-CSCs into CSCs (43). STAT3 regulates ALDH1 expression in cancer cells by enhancing the binding of transcription factor CCAAT enhance binding proteins $\beta(\mathrm{CEBP} / \mathrm{B})$ with ALDH1 promoter region $(44,45)$. Thus, targeting STAT3 activation-induced ALDH1A1 expression to eradicate OCSC has potential as a therapeutic strategy. These findings further support the possibility that inhibiting IL-6-induced activation of STAT3 could provide a means of targeting CSCs in OC. Anti-IL-6 antibody effectively blocks STAT3 activation by IL-6; however, IL-6-Nab treatment alone has not proven to be an effective therapeutic approach for advanced OC, suggesting that combinations with other targeted agents is necessary (27).

Epigenetic alterations play an important role in OCSC and OC chemotherapy resistance, being largely related to repression of tumor-suppressor genes (specifically, chemotherapy-response and differentiation-associated genes) by DNA methylation $(4,46,47)$. HMAs reverse the expression of silenced tumor suppressors and chemosensitize platinum-resistant ovarian tumors, resulting in significant clinical benefit (47-50). Importantly, epigenetic therapies, especially HMAs, have been demonstrated to reactivate epigenetic silencing in cancer immunotherapy, remove $\mathrm{T}$ cell repression, increase effector $\mathrm{T}$ cell tumor infiltration, inhibit tumor growth, and improve therapeutic efficacy of immunotherapy, such as anti-PD-1/PD-L1 $(51,52)$ and NY-ESO-1 vaccine therapy (53). We have previously shown that $\mathrm{ALDH}^{+} \mathrm{OCSC}$ harbor aberrant DNMT signatures and examined the effects of low-dose guadecitabine on OCSC. We found that this HMA reduces the OCSC population by driving these cells toward differentiation (4). Those results and the previous findings that blockade of the IL-6/STAT3 signaling pathway induced apoptosis and resensitized OC cells to chemotherapy $(30,54,55)$ support a combination strategy between an HMA and a STAT3 inhibitor, as we tested here.

The expression of ALDH isoforms, which are responsible for the aldefluor activity, is cell type dependent. Our group and others have shown the expression of ALDH1A1 isoform is correlated to enzymatic activity in the majority of OC cells $(31,56)$. However, this does not appear to be the case for some OC cells, including OVCAR4, as the dominant isoform for ALDH enzymatic activity in this cell lines is not 
ALDH1A1 but, instead, other ALDH1 isoforms (ALDH1A2 and ALDH1A3). Although there was no correlation between expression of IL-6 and IL-6R with ALDH1A1 expression in OVCAR4 cells, IL-6 and IL-6R significantly correlated with ALDH enzymatic activity (Supplemental Figure 1A), which determines stemness phenotypes. However, transcription factor CEBP/B binding sites are present in the promoter region of ALDH1 isoform genes, indicating a similar mechanism of regulation.

It has also been widely demonstrated that STAT3 regulates ALDH1 expression in cancer cells by enhancing the binding of transcription factor CEBP/B with the ALDH1 promoter region $(44,45)$. Moreover, in Figure 2B, we show that IL-6/IL-6R expression is higher in OVCAR4-derived ALDH ${ }^{+}$cells compared with $\mathrm{ALDH}^{-}$cells, which is consistent with our conclusion that expression of IL-6/IL-6 R correlates with stemness phenotypes, detected by aldefuor activity, in OC cells.

Here, we show that the combination of guadecitabine with IL-6-Nab was more effective than either treatment alone at preventing tumor relapse after platinum therapy. IL-6-Nab alone had no significant impact on recurrent tumor growth, but IL-6-Nab-guadecitabine effectively decreased the $\mathrm{ALDH}^{+} \mathrm{OCSC}$ population. Interestingly, the anti-OCSC effects of IL-6-Nab were more apparent in vivo than in the 2-dimensional culture OC cells, supporting a combined effect on cancer cell and on components of the TME. These results strongly support future clinical testing of this combination as a posttreatment strategy in OC.

In conclusion, we demonstrate that IL- 6 secreted by fibroblasts after platinum treatment promotes enrichment of OCSC. Maintenance treatment with IL-6-Nab-guadecitabine after carboplatin inhibited OCSC enrichment by both inhibiting DNMT expression and blocking pSTAT3-mediated ALDH1A1 transcription. The significance of the study lies in the concept of a potentially novel maintenance therapy in platinum-treated tumor residuals and recurrent tumors, based on the anti-OCSC effect of this combination therapy. These results propose a new combination of IL-6-Nab and epigenetic therapy as a maintenance strategy after chemotherapy for eradicating OCSC and preventing tumor recurrence.

\section{Methods}

Cell lines, culture conditions, and reagents. Human epithelial OC cell lines were maintained in the indicated media (Supplemental Table 3) at $30^{\circ} \mathrm{C}$ and $5 \% \mathrm{CO}_{2}$. NOFs were isolated from omentum of noncancer patients and maintained in DMEM/F12 with 1\% MEM vitamins, 1\% MEM nonessential amino acids, and 1\% penicillin-streptomycin. All cell lines were authenticated in 2017 by ATCC and tested for mycoplasma contamination. Reagents are described in Supplemental Methods.

Aldefluor assay and flow cytometry. ALDH1 enzymatic activity was measured using the Aldefluor assay kit (Stemcell Technologies) following the manufacturer's instructions, as described in our previous study (4).

Quantitative PCR ( $q P C R$ ). Cultured cells were directly lysed in the RLT buffer (RNeasy Lysis Buffer, Qiagen). RNA was isolated by using AllPrep DNA/RNA/Protein Mini kit (Qiagen) following the manufacturer's protocol. cDNA was transcribed from total RNA (from $100 \mathrm{ng}-2.5 \mu \mathrm{g}$ ) by using SuperScript VILO cDNA synthesis kit (catalog 11754-010, Thermo Fisher Scientific), following the manufacturer's instructions. qPCR was performed using LightCycler 480 SYBR Green I Master kit (Roche Diagnostics), and mRNA expression level was determined using LightCycler software version 3.5 (Roche Applied Science) and normalized to EEF1A. Primer sequences for qPCR (Thermo Fisher Scientific) can be found in Supplemental Table 4.

Clonogenic survival assay. Colony formation assay was performed on OC cells, as described in Supplemental Methods.

Transfection assays. Kuramochi and A2780 cells were seeded and grown into 6-cm well plates to 70\% confluence in RPMI media supplemented with $10 \%$ FBS; they were then transfected with shALDH1A1 plasmid DNA, shIL-6 plasmid DNA (6 $\mu$ g, Santa Cruz Biotechnology Inc.), or scrambled control DNA oligos ( $6 \mu \mathrm{g})$ for 48 hours using Turbofect (Thermo Fisher Scientific), according to manufacturer's protocol. Single, stably transfected colonies were selected by using puromycin antibiotic.

Human IL-6 ELISA and cytokine release assays. Cells were washed with PBS and then cultured in serum-starving media with $0.5 \%$ FBS prior to any treatment. CM was collected at indicated time points after treatment withdrawal. Cells were also collected, and the total number of cells was determined. IL- 6 secretion levels in CM were measured by using human IL-6 ELISA Ready-SET-Go kits eBiosciences (catalog 88-7066-86) and normalized to the cell number $\left(\mathrm{pg} / \mathrm{ml} / 1 \times 10^{5}\right.$ cells). Global cytokine expression in CM was measured by using cytokine release assay (R\&D Systems, catalog ARY005).

Spheroid formation assay. Spheroid formation assay was performed on OC cells and xenogtaft disassociated tumors, as described in the Supplemental Methods. 
Western blotting. Cells were lysed in RIPA lysis buffer (50 mM Tris- $\mathrm{HCl}, \mathrm{pH} 8.0,150 \mathrm{mM} \mathrm{NaCl}, 1 \mathrm{mM}$ EDTA, 1\% NP-40, 0.5\% sodium deoxycholate, and 0.1\% SDS) supplemented with (1×) protease inhibitors (MilliporeSigma). Xenograft tumors were lysed in RIPA buffer with protease inhibitors and homogenized using Rino tissue homogenization beads lysis kits as described above. Protein was isolated subject to Western blot as described (18). Antibodies used for Western blotting are described in Supplemental Methods.

In vivo xenograft experiments. Athymic, BALB/c-nu/nu mice (Female nude; 6 weeks old; Harlan) were injected i.p. with $2 \times 10^{6}$ (A2780) cells. Three days after injection, mice were i.p. treated with carboplatin (MilliporeSigma) at $50 \mathrm{mg} / \mathrm{kg}$, or PBS ( $n=6$ animals per group) weekly for 3 weeks. For the maintenance study, after 3 weeks of carboplatin treatment, mice were randomized to receive guadecitabine $(2 \mathrm{mg} / \mathrm{kg})$ s.c., mouse IgG (5 mg/ml, InvivoGen), IL-6-Nab (IL-6-Nab; $5 \mathrm{mg} / \mathrm{kg}$, InvivoGen) (i.p.) alone or in combination with guadecitabine, or vehicle (diluent) twice a week for 2 weeks. Mice were euthanized, and peritoneal tumors were counted and weighed at the end of the study. Tumors and blood samples were processed as described in Supplemental Methods.

Statistics. All experiments were performed in triplicate, and mean values \pm SD of biological triplicate measurements were calculated. Western blots were performed with biological duplicates, and representative images are presented in the figures. Two-tailed Student's $t$ test was used to statistically analyze the significant difference between 2 groups by using Prism 4.0 (GraphPad Software), with a $P$ value of 0.05 being considered statistically significant. One-way and 2-way ANOVA including Dunnett's and Sidak's multiple comparisons tests were used for multiple comparisons by using Prism 4.0, as well. A $P$ value of 0.05 was considered statistically significant.

Study approval. All animal studies adhered to ethical regulations and protocols approved by the IACUC of Indiana University (Indianapolis, Indiana, USA). The IRB of Indiana University approved this research, and informed consent was obtained from all patients.

\section{Author contributions}

YW designed the research, performed the experiments, analyzed results and wrote the manuscript. XZ reviewed the manuscript and performed and analyzed some of the experiments. SM and AKM designed some of the experiments, analyzed the data, and reviewed the manuscript. DM reviewed the manuscript. KPN designed the research, analyzed results, and contributed to writing of the manuscript.

\section{Acknowledgments}

We thank Harold N. Keer (Astex Pharmaceuticals Inc.) for providing guadecitabine for research use in this study. We thank Pietro Taverna (Sunesis Pharmaceuticals) for helpful discussions and Fang Fang (Indiana University School of Medicine) and Nwani Nkechiyere (Northwestern University Feinberg School of Medicine) for help with manuscript preparation. We recognize the use of the IUB Flow Cytometry Core Facility and thank Christiane Hassel for FACS sorting and analyzing samples. We thank Fang Fang for technical support. This work was funded by a Collaborative Research Development grant from the Ovarian Cancer Research Fund Alliance.

Address correspondence to: Kenneth P. Nephew, Indiana University School of Medicine, Medical Sciences, Jordan Hall 302, 1001 E Third Street, Bloomington, Indiana 47405-4401, USA. Phone: 812.855.9445; Email: knephew@indiana.edu.

1. Berek JS, et al. Advanced epithelial ovarian cancer: 1998 consensus statements. Ann Oncol. 1999;10 Suppl 1:87-92.

2. Bowtell DD, et al. Rethinking ovarian cancer II: reducing mortality from high-grade serous ovarian cancer. Nat Rev Cancer. 2015;15(11):668-679.

3. Dean M, Fojo T, Bates S. Tumour stem cells and drug resistance. Nat Rev Cancer. 2005;5(4):275-284.

4. Wang Y, et al. Epigenetic targeting of ovarian cancer stem cells. Cancer Res. 2014;74(17):4922-4936.

5. Zhang S, et al. Identification and characterization of ovarian cancer-initiating cells from primary human tumors. Cancer Res. 2008;68(11):4311-4320.

6. Silva IA, et al. Aldehyde dehydrogenase in combination with CD133 defines angiogenic ovarian cancer stem cells that portend poor patient survival. Cancer Res. 2011;71(11):3991-4001.

7. Nguyen LV, Vanner R, Dirks P, Eaves CJ. Cancer stem cells: an evolving concept. Nat Rev Cancer. 2012;12(2):133-143.

8. Marcato P, et al. Aldehyde dehydrogenase activity of breast cancer stem cells is primarily due to isoform ALDH1A3 and its expression is predictive of metastasis. Stem Cells. 2011;29(1):32-45.

9. Kastan MB, Schlaffer E, Russo JE, Colvin OM, Civin CI, Hilton J. Direct demonstration of elevated aldehyde dehydrogenase in 
human hematopoietic progenitor cells. Blood. 1990;75(10):1947-1950.

10. Chaffer CL, et al. Poised chromatin at the ZEB1 promoter enables breast cancer cell plasticity and enhances tumorigenicity. Cell. 2013;154(1):61-74.

11. Wang D, et al. Autocrine interleukin-23 promotes self-renewal of CD133+ ovarian cancer stem-like cells. Oncotarget 2016;7(46):76006-76020.

12. Li J, et al. Lipid Desaturation Is a Metabolic Marker and Therapeutic Target of Ovarian Cancer Stem Cells. Cell Stem Cell. 2017;20(3):303-314.e5.

13. Rojas A, et al. IL-6 promotes prostate tumorigenesis and progression through autocrine cross-activation of IGF-IR. Oncogene. 2011;30(20):2345-2355.

14. Hodge DR, Hurt EM, Farrar WL. The role of IL-6 and STAT3 in inflammation and cancer. Eur J Cancer. 2005;41(16):2502-2512.

15. Studebaker AW, et al. Fibroblasts isolated from common sites of breast cancer metastasis enhance cancer cell growth rates and invasiveness in an interleukin-6-dependent manner. Cancer Res. 2008;68(21):9087-9095.

16. Tao L, Huang G, Song H, Chen Y, Chen L. Cancer associated fibroblasts: An essential role in the tumor microenvironment. Oncol Lett. 2017;14(3):2611-2620.

17. Mitra AK, et al. MicroRNAs reprogram normal fibroblasts into cancer-associated fibroblasts in ovarian cancer. Cancer Discov. 2012;2(12):1100-1108.

18. Özeş AR, et al. NF-kB-HOTAIR axis links DNA damage response, chemoresistance and cellular senescence in ovarian cancer. Oncogene. 2016;35(41):5350-5361.

19. Garcia-Gomez A, Rodríguez-Ubreva J, Ballestar E. Epigenetic interplay between immune, stromal cancer cells in the tumor microenvironment [published online ahead of print March 6, 2018]. Clin Immunol. https://doi.org/10.1016/j.clim.2018.02.013.

20. Lin HJ, et al. Breast cancer-associated fibroblasts confer AKT1-mediated epigenetic silencing of Cystatin M in epithelial cells. Cancer Res. 2008;68(24):10257-10266.

21. Pidsley R, et al. Enduring epigenetic landmarks define the cancer microenvironment. Genome Res. 2018;28(5):625-638.

22. Pistore $\mathrm{C}$, et al. DNA methylation variations are required for epithelial-to-mesenchymal transition induced by cancer-associated fibroblasts in prostate cancer cells. Oncogene. 2017;36(40):5551-5566.

23. Pasquier J, Rafii A. Role of the microenvironment in ovarian cancer stem cell maintenance. Biomed Res Int. 2013;2013:630782.

24. Foran E, et al. Upregulation of DNA methyltransferase-mediated gene silencing, anchorage-independent growth, and migration of colon cancer cells by interleukin-6. Mol Cancer Res. 2010;8(4):471-481.

25. Gasche JA, Hoffmann J, Boland CR, Goel A. Interleukin-6 promotes tumorigenesis by altering DNA methylation in oral cancer cells. Int J Cancer. 2011;129(5):1053-1063.

26. Guo Y, et al. Effects of siltuximab on the IL-6-induced signaling pathway in ovarian cancer. Clin Cancer Res. 2010;16(23):5759-5769.

27. Milagre CS, et al. Adaptive Upregulation of EGFR Limits Attenuation of Tumor Growth by Neutralizing IL6 Antibodies, with Implications for Combined Therapy in Ovarian Cancer. Cancer Res. 2015;75(7):1255-1264.

28. Landen CN, et al. Targeting aldehyde dehydrogenase cancer stem cells in ovarian cancer. Mol Cancer Ther. 2010;9(12):3186-3199.

29. Cohen S, et al. Platinum-resistance in ovarian cancer cells is mediated by IL-6 secretion via the increased expression of its target cIAP-2. J Mol Med. 2013;91(3):357-368.

30. Wang ZY, et al. IL-6 Inhibition Reduces STAT3 Activation and Enhances the Antitumor Effect of Carboplatin. Mediators Inflamm. 2016;2016:8026494.

31. Meng E, et al. ALDH1A1 maintains ovarian cancer stem cell-like properties by altered regulation of cell cycle checkpoint and DNA repair network signaling. PLoS One. 2014;9(9):e107142.

32. McLean K, et al. Leukemia inhibitory factor functions in parallel with interleukin-6 to promote ovarian cancer growth [published online ahead of print October 10, 2018]. Oncogene. https://doi.org/10.1038/s41388-018-0523-6.

33. Chu Y, et al. Adipose-derived mesenchymal stem cells promote cell proliferation and invasion of epithelial ovarian cancer. Exp Cell Res. 2015;337(1):16-27.

34. Smith AK, et al. Epigenetic changes associated with inflammation in breast cancer patients treated with chemotherapy. Brain Behav Immun. 2014;38:227-236.

35. Wintzell M, Löfstedt L, Johansson J, Pedersen AB, Fuxe J, Shoshan M. Repeated cisplatin treatment can lead to a multiresistant tumor cell population with stem cell features and sensitivity to 3-bromopyruvate. Cancer Biol Ther. 2012;13(14):1454-1462

36. Özeş AR, et al. NF-kB-HOTAIR axis links DNA damage response, chemoresistance and cellular senescence in ovarian cancer. Oncogene. 2016;35(41):5350-5361.

37. Paschall AV, Liu KB. Epigenetic and Immune Regulation of Colorectal Cancer Stem Cells. Curr Colorect Canc R. 2015;11(6):414-421.

38. Zou W, Wicha MS. Chemokines and cellular plasticity of ovarian cancer stem cells. Oncoscience. 2015;2(7):615-616.

39. Peng D, et al. Myeloid-Derived Suppressor Cells Endow Stem-like Qualities to Breast Cancer Cells through IL6/STAT3 and NO/NOTCH Cross-talk Signaling. Cancer Res. 2016;76(11):3156-3165.

40. Han Z, et al. Inhibition of STAT3 signaling targets both tumor-initiating and differentiated cell populations in prostate cancer. Oncotarget. 2014;5(18):8416-8428.

41. Lin L, et al. STAT3 is necessary for proliferation and survival in colon cancer-initiating cells. Cancer Res. 2011;71(23):7226-7237.

42. Lin L, et al. Evaluation of STAT3 signaling in ALDH+ and ALDH+/CD44+/CD24- subpopulations of breast cancer cells. PLoS ONE. 2013;8(12):e82821.

43. Kim RJ, et al. High aldehyde dehydrogenase activity enhances stem cell features in breast cancer cells by activating hypoxia-inducible factor-2 $\alpha$. Cancer Lett. 2013;333(1):18-31.

44. Canino C, Luo Y, Marcato P, Blandino G, Pass HI, Cioce M. A STAT3-NFkB/DDIT3/CEBP $\beta$ axis modulates ALDH1A3 expression in chemoresistant cell subpopulations. Oncotarget. 2015;6(14):12637-12653.

45. Alam M, Ahmad R, Rajabi H, Kharbanda A, Kufe D. MUC1-C oncoprotein activates ERK $\rightarrow$ C/EBP $\beta$ signaling and induction of aldehyde dehydrogenase 1A1 in breast cancer cells. J Biol Chem. 2013;288(43):30892-30903.

46. Fang F, et al. A phase 1 and pharmacodynamic study of decitabine in combination with carboplatin in patients with recurrent, platinum-resistant, epithelial ovarian cancer. Cancer. 2010;116(17):4043-4053.

47. Matei D, et al. Epigenetic resensitization to platinum in ovarian cancer. Cancer Res. 2012;72(9):2197-2205. 
48. Fang F, et al. Decitabine reactivated pathways in platinum resistant ovarian cancer. Oncotarget. 2014;5(11):3579-3589.

49. Fang F, et al. The novel, small-molecule DNA methylation inhibitor SGI-110 as an ovarian cancer chemosensitizer. Clin Cancer Res. 2014;20(24):6504-6516.

50. Fang F, et al. Genomic and Epigenomic Signatures in Ovarian Cancer Associated with Resensitization to Platinum Drugs. Cancer Res. 2018;78(3):631-644.

51. Peng D, et al. Epigenetic silencing of TH1-type chemokines shapes tumour immunity and immunotherapy. Nature 2015;527(7577):249-253.

52. Ørskov AD, et al. Hypomethylation and up-regulation of PD-1 in T cells by azacytidine in MDS/AML patients: A rationale for combined targeting of PD-1 and DNA methylation. Oncotarget. 2015;6(11):9612-9626.

53. Odunsi K, et al. Epigenetic potentiation of NY-ESO-1 vaccine therapy in human ovarian cancer. Cancer Immunol Res. 2014;2(1):37-49.

54. Han Z, et al. Silencing of the STAT3 signaling pathway reverses the inherent and induced chemoresistance of human ovarian cancer cells. Biochem Biophys Res Commun. 2013;435(2):188-194.

55. Pu YS, Hour TC, Chuang SE, Cheng AL, Lai MK, Kuo ML. Interleukin-6 is responsible for drug resistance and anti-apoptotic effects in prostatic cancer cells. Prostate. 2004;60(2):120-129.

56. Condello S, et al. $\beta$-Catenin-regulated ALDH1A1 is a target in ovarian cancer spheroids. Oncogene. 2015;34(18):2297-2308. 